\title{
Electric and magnetic dipole strength in ${ }^{66} \mathrm{Zn}$
}

\author{
R. Schwengner ${ }^{1}{ }^{1}$ R. Massarczyk, ${ }^{2}$ M. Scheck, ${ }^{3}$ W. Tornow, ${ }^{4,5}$ G. Battaglia, ${ }^{6}$ T. Beck, ${ }^{7}$ D. Bemmerer, ${ }^{1}$ N. Benouaret, ${ }^{8}$ \\ R. Beyer, ${ }^{1}$ M. Butterling, ${ }^{1}$ F. Fiedler, ${ }^{1}$ S. W. Finch, ${ }^{4,5}$ C. Fransen, ${ }^{9}$ U. Friman-Gayer, ${ }^{5,10}$ A. Frotscher, ${ }^{1,}{ }^{*}$ R. Gonzalez, ${ }^{5,10}$ \\ M. Grieger, ${ }^{1,11}$ A. Hartmann, ${ }^{1}$ T. Hensel, ${ }^{1,11}$ E. Hoemann, ${ }^{9}$ H. Hoffmann, ${ }^{1}$ R. V. F. Janssens, ${ }^{5,10}$ S. Johnson, ${ }^{5,10}$ \\ M. D. Jones, ${ }^{5,10}$ A. R. Junghans, ${ }^{1}$ N. Kelly, ${ }^{3}$ J. Kleemann, ${ }^{7}$ Krishichayan, ${ }^{4,5}$ D. R. Little, ${ }^{10}$ F. Ludwig, ${ }^{1,11}$ S. E. Müller, ${ }^{1}$ \\ D. O’Donnell, ${ }^{3}$ O. Papst, ${ }^{7}$ E. Pirovano, ${ }^{1, \dagger}$ J. Sinclair, ${ }^{3}$ M. P. Takács, ${ }^{1, \dagger}$ S. Turkat, ${ }^{11}$ S. Urlaß, ${ }^{1,12}$ A. Wagner, ${ }^{1}$ V. Werner, ${ }^{7}$ \\ O. Wieland, ${ }^{13}$ and J. Wilhelmy ${ }^{9}$ \\ ${ }^{1}$ Helmholtz-Zentrum Dresden-Rossendorf, 01328 Dresden, Germany \\ ${ }^{2}$ Los Alamos National Laboratory, Los Alamos, New Mexico 87545, USA \\ ${ }^{3}$ University of the West of Scotland, Paisley PA1 2BE, United Kingdom \\ ${ }^{4}$ Department of Physics, Duke University, Durham, North Carolina 27708, USA \\ ${ }^{5}$ Triangle Universities Nuclear Laboratory, Durham, North Carolina 27708, USA \\ ${ }^{6}$ University of Strathclyde, Glasgow G4 ONG, United Kingdom \\ ${ }^{7}$ Institut für Kernphysik, Technische Universität Darmstadt, 64289 Darmstadt, Germany \\ ${ }^{8}$ Faculté de Physique, Université des Sciences et de la Technologie d'Alger (USTHB), El-Alia 16111, Bab-Ezzouar-Alger, Algerie \\ ${ }^{9}$ Institut für Kernphysik, Universität zu Köln, 50937 Köln, Germany \\ ${ }^{10}$ Department of Physics and Astronomy, University of North Carolina at Chapel Hill, Chapel Hill, North Carolina 27599, USA \\ ${ }^{11}$ Institut für Kern- und Teilchenphysik, Technische Universität Dresden, 01069 Dresden, Germany \\ ${ }^{12}$ European Organization for Nuclear Research (CERN), 1211 Meyrin, Geneva, Switzerland \\ ${ }^{13}$ INFN, Sezione di Milano, 20133 Milano, Italy
}

(Received 11 August 2020; accepted 1 February 2021; published 11 February 2021)

\begin{abstract}
The dipole strength of the nuclide ${ }^{66} \mathrm{Zn}$ was studied in photon-scattering experiments using bremsstrahlung produced with electron beams of energies of 7.5 and $13.4 \mathrm{MeV}$ at the $\gamma$ ELBE facility as well as using quasimonoenergetic and linearly polarized photon beams of 30 energies within the range of 4.3 to $9.9 \mathrm{MeV}$ at the $\mathrm{HI} \gamma \mathrm{S}$ facility. A total of $128 \mathrm{~J}=1$ states were identified, among them 9 with $1^{+}$and 86 with $1^{-}$ assignments. The quasicontinuum of unresolved transitions was included in the analysis of the spectra and the intensities of branching transitions were estimated on the basis of simulations of statistical $\gamma$-ray cascades. As a result, the photoabsorption cross section up to the neutron-separation energy was determined and compared with predictions of the statistical reaction model. The experimental $M 1$ strengths from resolved $1^{+}$states are compared with results of large-scale shell-model calculations.
\end{abstract}

DOI: 10.1103/PhysRevC.103.024312

\section{INTRODUCTION}

Experimental and theoretical studies of photon strength functions have attracted growing interest in the past years $[1,2]$. These functions describe average transition strengths in the energy region of the quasicontinuum of nuclear levels and are important ingredients for calculations of reaction cross sections within the statistical reaction model [3]. Such calculations are used, for example, to obtain information about radiative neutron-capture cross sections of unstable nuclides

\footnotetext{
*Present address: Institut für Kernphysik, Technische Universität Darmstadt, 64289 Darmstadt, Germany.

${ }^{\dagger}$ Present address: Physikalisch-Technische Bundesanstalt, 38116 Braunschweig, Germany.

Published by the American Physical Society under the terms of the Creative Commons Attribution 4.0 International license. Further distribution of this work must maintain attribution to the author(s) and the published article's title, journal citation, and DOI.
}

for modeling the astrophysical $s$ process [4,5]. Experimental photon strength functions are determined by using various nuclear reactions, such as light-ion induced reactions, neutron capture $(n, \gamma)$, proton scattering $\left(p, p^{\prime}\right)$, and photon scattering $\left(\gamma, \gamma^{\prime}\right)$. In the latter, also called nuclear resonance fluorescence (NRF), angular momentum $L=1$ is preferentially transferred by the incident photons and, for example in even-even nuclei, mainly states with spin and parity quantum numbers $J^{\pi}=1^{+}$and $1^{-}$are excited [6]. Energy-integrated scattering cross sections of these states and the photoabsorption cross section are determined from the intensities of the respective $\gamma$ transitions back to the ground state. In this case, the transition energy in the strength function $f\left(E_{\gamma}\right)$ is identical to the excitation energy in the photoabsorption cross section $\sigma_{\gamma}$ and the two quantities are connected via the relation $\sigma_{\gamma}=g(\pi \hbar c)^{2} E_{\gamma} f\left(E_{\gamma}\right)$ with $g=\left(2 J_{x}+1\right) /\left(2 J_{0}+1\right)$, where $J_{x}$ and $J_{0}$ are the spins of the excited and ground states, respectively.

The standard electric dipole $(E 1)$ strength functions used in statistical-reaction-model calculations are Lorentz functions 
adjusted to $(\gamma, n)$ reaction data that represent the isovector giant dipole resonance (GDR). Below the neutron-separation energy $S_{n}$, several additional structures have been found on top of the low-energy tail of the GDR. Just below $S_{n}$, a pygmy dipole resonance (PDR) appears [7-9], which is characterized by intense $E 1$ transitions and develops with increasing neutron excess [10]. The magnetic dipole $(M 1)$ spin-flip resonance evolves at around $8 \mathrm{MeV}$ and the $M 1$ scissors mode evolves around $3 \mathrm{MeV}$ in deformed nuclei [11]. These structures manifest their presence, for example, in $J^{\pi}=1^{+}$states excited from the $0^{+}$ground state. However, according to the Brink-Axel hypothesis [12,13], they can also be built on excited states. Indeed, resonance-like structures at energies of the scissors mode have been observed in strength functions obtained from light-ion induced reactions that include many transitions between excited states of various spins [14-16]. A surprising feature in the shape of the dipole strength function is an upbend toward low energies $\left(E_{\gamma} \lesssim 2 \mathrm{MeV}\right)$, which has been observed in several nuclides using light-ion induced reactions, for example in ${ }^{56,57} \mathrm{Fe}[17,18]$, in Mo isotopes [19], in ${ }^{105,106} \mathrm{Cd}$ [20], and in Sm isotopes $[15,16]$. This low-energy enhancement of dipole strength has been described in shellmodel calculations as being caused by large $B(M 1)$ transition strengths of transitions linking close-lying states of various spins at high excitation energy [21-24]. For the $J=1$ states populated in $\left(\gamma, \gamma^{\prime}\right)$ experiments, this effect is also expected, but difficult to observe because the low-energy $\gamma$ rays have small intensities compared to those of the ground-state transitions and are usually hidden in the huge background of photons scattered by atomic interactions with the target material [25]. However, the usual goal of $\left(\gamma, \gamma^{\prime}\right)$ experiments is the determination of scattering and absorption cross sections that are derived from the intensities of ground-state transitions.

In recent photon-scattering studies using broad-band bremsstrahlung at the $\gamma$ ELBE facility [26] of the HelmholtzZentrum Dresden-Rossendorf (HZDR), Germany, as well as using quasimonoenergetic, polarized $\gamma$ rays produced at the high-intensity $\gamma$-ray source $(\mathrm{HI} \gamma \mathrm{S})$ [27], operated by the Triangle Universities Nuclear Laboratory (TUNL) in Durham, North Carolina, USA, we observed enhanced $E 1$ strength in the PDR region from about 6 to $10 \mathrm{MeV}$, for example in nuclides around $N=50$ [28-30], in nuclides below $N=82$ $[10,31]$, in ${ }^{181} \mathrm{Ta}[32]$, and in $\mathrm{Pb}$ isotopes $[33,34]$. To investigate the possible influence of the PDR on cross sections and reaction rates, the experimental strength distributions were used as an input for the statistical reaction model [35,36]. The experiments at $\mathrm{HI} \gamma \mathrm{S}$ using polarized photon beams allow a distinction between the $E 1$ and $M 1$ contributions to the photoabsorption cross section. An investigation of ${ }^{128} \mathrm{Xe}$ and ${ }^{134} \mathrm{Xe}$ including strength in the quasicontinuum of unresolved states proved that the main part $(\approx 90 \%)$ of the photoabsorption cross section in the PDR region is of $E 1$ character whereas the $M 1$ cross section gives only a relatively small contribution [37]. This relation seems to change when going to lighter nuclides, such as the ones in the iron-nickel region. In ${ }^{50} \mathrm{Cr}[38],{ }^{52} \mathrm{Cr}[39-41],{ }^{56} \mathrm{Fe}[42,43]$, and ${ }^{58,60} \mathrm{Ni}$ $[42,44,45]$, several strong isolated $M 1$ excitations have been observed. To study the relation between $E 1$ and $M 1$ strengths in this mass region for varying properties such as nuclear deformation, we studied a closed-shell nuclide, ${ }^{54} \mathrm{Fe}$, and an open-shell nuclide, ${ }^{66} \mathrm{Zn}$. While the results for ${ }^{54} \mathrm{Fe}$ were published elsewhere [46], we report the results for ${ }^{66} \mathrm{Zn}$ in this work.

In an earlier NRF experiment on ${ }^{66} \mathrm{Zn}$, seven $J=1$ states were observed at excitation energies between 3.3 and 4.8 $\mathrm{MeV}$ [47]. In another experiment, the photoexcitation of the $7368 \mathrm{keV}$ level by capture $\gamma$ rays from lead was studied [51]. We performed experiments at $\gamma$ ELBE using electron energies of 7.5 and $13.4 \mathrm{MeV}$ to determine integrated scattering cross sections and spins of excited states and the photoabsorption cross section including contributions from the quasicontinuum of states up to $S_{n}=11.1 \mathrm{MeV}$, and experiments at $\mathrm{HI} \gamma \mathrm{S}$ using photon beams of energies from 4.3 to $9.9 \mathrm{MeV}$ to determine the parities of the excited states.

\section{EXPERIMENTAL METHODS AND RESULTS}

\section{A. The photon-scattering method}

In photon-scattering experiments, the energy- and solidangle-integrated scattering cross section $I_{S}$ of an excited state at the energy $E_{x}$ is deduced from the measured intensity of the respective transition to the ground state. It can be determined relative to known integrated scattering cross sections. In the present experiments, we used the integrated scattering cross sections $I_{s}\left(E_{x}^{\mathrm{B}}\right)$ of states in ${ }^{11} \mathrm{~B}[52]$ and their angular correlations including mixing ratios [53] as a reference:

$$
\begin{aligned}
\frac{I_{s}\left(E_{x}\right)}{I_{s}\left(E_{x}^{\mathrm{B}}\right)}= & \left(\frac{I_{\gamma}\left(E_{\gamma}, \theta\right)}{W\left(E_{\gamma}, \theta\right) \Phi_{\gamma}\left(E_{x}\right) N_{N}}\right) \\
& \times\left(\frac{I_{\gamma}\left(E_{\gamma}^{\mathrm{B}}, \theta\right)}{W\left(E_{\gamma}^{\mathrm{B}}, \theta\right) \Phi_{\gamma}\left(E_{x}^{\mathrm{B}}\right) N_{N}^{\mathrm{B}}}\right)^{-1} .
\end{aligned}
$$

Here, $I_{\gamma}\left(E_{\gamma}, \theta\right)$ and $I_{\gamma}\left(E_{\gamma}^{\mathrm{B}}, \theta\right)$ denote the efficiency-corrected measured intensities of a considered ground-state transition at energy $E_{\gamma}$ and of a ground-state transition in ${ }^{11} \mathrm{~B}$ at $E_{\gamma}^{\mathrm{B}}$, respectively, observed at an angle $\theta$ to the beam. $W\left(E_{\gamma}, \theta\right)$ and $W\left(E_{\gamma}^{\mathrm{B}}, \theta\right)$ describe the angular correlations of these transitions. The quantities $N_{N}$ and $N_{N}^{\mathrm{B}}$ are the areal densities of nuclei in the ${ }^{66} \mathrm{Zn}$ and ${ }^{11} \mathrm{~B}$ targets, respectively. The quantities $\Phi_{\gamma}\left(E_{x}\right)$ and $\Phi_{\gamma}\left(E_{x}^{\mathrm{B}}\right)$ stand for the photon fluxes at the energy of the considered level and at the energy of a level in ${ }^{11} \mathrm{~B}$, respectively.

The integrated scattering cross section is related to the partial width of the ground-state transition $\Gamma_{0}$ according to

$$
I_{s}=\int_{0}^{+\infty} \sigma_{\gamma \gamma} d E=\left(\frac{\pi \hbar c}{E_{x}}\right)^{2} \frac{2 J_{x}+1}{2 J_{0}+1} \frac{\Gamma_{0}^{2}}{\Gamma},
$$

where $\sigma_{\gamma \gamma}$ is the elastic scattering cross section, $E_{x}, J_{x}$, and $\Gamma$ denote energy, spin and total width of the excited level, respectively, and $J_{0}$ is the spin of the ground state. If a given level deexcites to low-lying excited states (inelastic scattering) in addition to the deexcitation to the ground state (elastic scattering), then the branching ratio $b_{0}=\Gamma_{0} / \Gamma$ of the ground-state transition has to be known to deduce $\Gamma_{0}$. The $\gamma$-ray intensities and, hence, the deduced quantities $I_{s}$ and $\Gamma_{0}$ are also distorted if a level is populated from higher-lying levels. This feeding 
can be reduced by choosing beam energies not far above the considered levels. In experiments with quasimonoenergetic photons, a few levels are excited in a narrow energy window and feeding of these levels is excluded.

Spins of excited states can be deduced by comparing experimental ratios of $\gamma$-ray intensities, measured at two angles, with theoretical predictions. The optimum combination includes angles of $90^{\circ}$ and $127^{\circ}$ to the beam direction, because the respective ratios for the spin sequences $0-1-0$ and 0-2-0 differ most at these angles. The expected values are $W\left(90^{\circ}\right) / W\left(127^{\circ}\right)_{0-1-0}=0.74$ and $W\left(90^{\circ}\right) / W\left(127^{\circ}\right)_{0-2-0}=$ 2.15 taking into account opening angles of $16^{\circ}$ and $14^{\circ}$ of the detectors placed at $90^{\circ}$ and $127^{\circ}$, respectively, in the setup at $\gamma$ ELBE [26].

Parity quantum numbers of excited states can be determined by measuring the linear polarizations of the groundstate transitions. These are deduced from experimental asymmetries of intensities measured parallel or perpendicular to the polarization plane of the incident photon beam in the experiments at $\mathrm{HI} \gamma \mathrm{S}$.

\section{B. The target}

The target consisted of $1.4993 \mathrm{~g}$ of zinc, enriched to $98.00 \%$ in ${ }^{66} \mathrm{Zn}$, and shaped to a disk of $20 \mathrm{~mm}$ in diameter. In the experiments at $\gamma$ ELBE, the target was combined with $0.300 \mathrm{~g}$ of boron, enriched to $99.5 \%$ in ${ }^{11} \mathrm{~B}$, and also formed to a disk of $20 \mathrm{~mm}$ in diameter. The known integrated scattering cross sections of levels in ${ }^{11} \mathrm{~B}$ were used to determine the photon flux.

\section{Detector response}

For the determination of the integrated scattering cross sections according to Eq. (1) the efficiencies of the detectors and the photon flux are needed. The detector response was simulated using the program package GEANT4 [54-56]. The reliability of the simulations was tested by comparing simulated spectra with measured ones as described, for example, in Refs. [57-61]. The determination of the absorption cross section requires in addition a correction of the experimental spectra for photons scattered by atomic processes induced by the impinging photons in the target material, and for ambient background radiation, which is described in Sec. III.

The absolute efficiencies of the high-purity germanium (HPGe) detectors in the setup at $\gamma$ ELBE were determined experimentally up to $2.4 \mathrm{MeV}$ from measurements with a ${ }^{226} \mathrm{Ra}$ calibration source. For interpolation, an efficiency curve calculated with GEANT4 and scaled to the absolute experimental values was used. A check of the simulated efficiency curve up to about $9 \mathrm{MeV}$ was performed via various $(p, \gamma)$ reactions at the HZDR Tandetron accelerator. The efficiency values deduced from these measurements agree with the simulated values within their uncertainties [62]. Similar results were obtained for the resonances at 4.44 and $11.66 \mathrm{MeV}$ in ${ }^{12} \mathrm{C}$ populated in the ${ }^{11} \mathrm{~B}(p, \gamma)$ reaction at the TUNL van de Graaff accelerator [63].

\section{Experiments with bremsstrahlung at $\gamma \mathrm{ELBE}$}

The nuclide ${ }^{66} \mathrm{Zn}$ was studied in two experiments at $\gamma$ ELBE [26]. Bremsstrahlung was produced using electron beams of 7.5 and $13.4 \mathrm{MeV}$ kinetic energy. In the measurement at $7.5 \mathrm{MeV}$, the electron beam hit a niobium foil of $5 \mu \mathrm{m}$ thickness acting as a radiator at an average current of about $670 \mu \mathrm{A}$. In the measurement at $13.4 \mathrm{MeV}$, the niobium foil had a thickness of $12.5 \mu \mathrm{m}$ and the average current was about $490 \mu \mathrm{A}$. A $10 \mathrm{~cm}$ thick aluminum absorber (beam hardener) was placed behind the radiator to reduce the low-energy intensity of the bremsstrahlung spectrum in the measurement at 13.4 MeV. The photon beam was collimated by a $260-\mathrm{cm}-\mathrm{long}$ pure-aluminum collimator with a conical borehole of $8 \mathrm{~mm}$ in diameter at the entrance, $90 \mathrm{~cm}$ behind the radiator, and $24 \mathrm{~mm}$ in diameter at the exit. The target, placed $200 \mathrm{~cm}$ behind the collimator exit, was irradiated with a typical flux of about $10^{9} \mathrm{~s}^{-1}$ in a spot of $38 \mathrm{~mm}$ in diameter. Scattered photons were measured with four high-purity germanium (HPGe) detectors with a full-energy efficiency of $100 \%$ relative to a $\mathrm{NaI}$ detector of $7.6 \mathrm{~cm}$ in diameter and $7.6 \mathrm{~cm}$ in length. All HPGe detectors were surrounded by escape-suppression shields made of bismuth germanate (BGO) scintillation detectors of $3 \mathrm{~cm}$ in thickness. Two HPGe detectors were placed vertically at $127^{\circ}$ relative to the photon-beam direction and a distance of $32 \mathrm{~cm}$ from the target. The other two HPGe detectors were positioned in a horizontal plane at $90^{\circ}$ to the beam and a distance of $28 \mathrm{~cm}$ from the target. Absorbers of $8 \mathrm{~mm} \mathrm{~Pb}$ plus $3 \mathrm{~mm} \mathrm{Cu}$ were placed in front of the detectors at $90^{\circ}$ and of $3 \mathrm{~mm} \mathrm{~Pb}$ plus $3 \mathrm{~mm} \mathrm{Cu}$ in front of the detectors at $127^{\circ}$. Spectra of scattered photons were measured for 74 and $120 \mathrm{~h}$ in the experiments at 7.5 and $13.4 \mathrm{MeV}$ electron energy, respectively. Part of a spectrum including events measured with the two detectors placed at $127^{\circ}$ relative to the beam at an electron energy of $13.4 \mathrm{MeV}$ is shown in Fig. 1.

The absolute photon fluxes in the two measurements at $\gamma$ ELBE were determined from intensities and known integrated scattering cross sections of transitions in ${ }^{11} \mathrm{~B}$. For interpolation, the photon flux was calculated using a bremsstrahlung computer code [64] based on the Born approximation with Coulomb correction [65] and including an atomic screening correction [66]. In addition, the flux was corrected for the attenuation by the beam hardener. The calculated flux curves were scaled to the experimental values obtained at the energies of levels in ${ }^{11} \mathrm{~B}$. The experimental flux values and the calculated curves are presented in Fig. 2.

The measurements at two electron energies allowed us to identify inelastic transitions feeding low-lying from highlying levels. Transitions found in the measurement at $E_{e}^{\mathrm{kin}}=$ 7.5 MeV are assumed to be ground-state transitions. Transitions additionally observed up to $7.5 \mathrm{MeV}$ in the measurement at $E_{e}^{\text {kin }}=13.4 \mathrm{MeV}$ are considered to be inelastic transitions from high-lying to low-lying excited states. By comparing the respective spectra, these inelastic transitions were sorted out. Besides, there is a number of transitions with energies that fit the difference between the energy of a higher-lying level and the first excited $2^{+}$state. These transitions are also assumed to be inelastic transitions, if their intensity is smaller than that of the ground-state transition from the considered higher-lying 


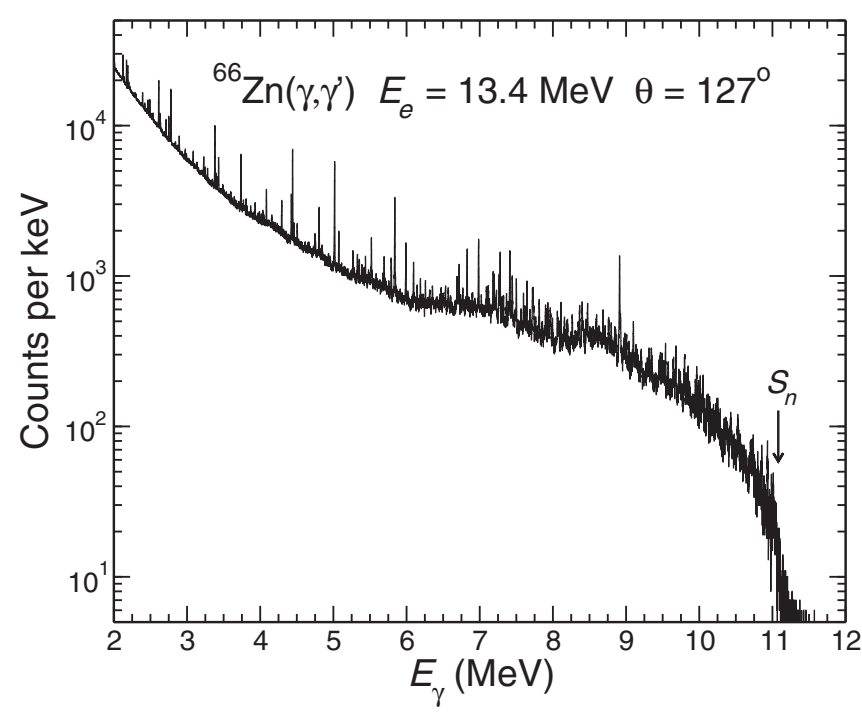

FIG. 1. Part of a spectrum of photons scattered from ${ }^{66} \mathrm{Zn}$ combined with ${ }^{11} \mathrm{~B}$, measured during the irradiation with bremsstrahlung produced by electrons of an energy of $E_{e}^{\mathrm{kin}}=13.4 \mathrm{MeV}$. This spectrum is the sum of the spectra measured with the two detectors placed at $127^{\circ}$ relative to the beam at $\gamma \mathrm{ELBE}$.

level. The remaining ground-state transitions were used to derive the corresponding level energies, the integrated scattering cross sections of the states, and spin assignments deduced from angular distributions of the ground-state transitions, that are compiled in Table I. The integrated scattering cross sections of levels up to $E_{x}=7.0 \mathrm{MeV}$ were taken from the measurement at $7.5 \mathrm{MeV}$ electron energy, because they are affected by feeding intensities in the $13.4 \mathrm{MeV}$ measurement. To illustrate this influence, values of the two measurements are compared with each other and with the results of previous

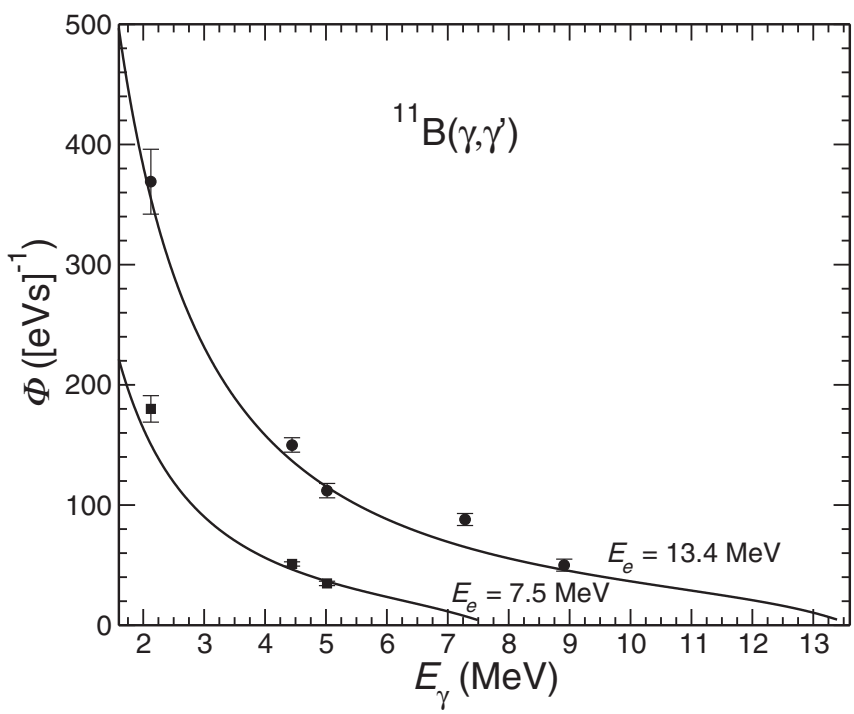

FIG. 2. Absolute photon flux on the target deduced from intensities of known transitions in ${ }^{11} \mathrm{~B}$ for the measurements at $E_{e}=$ 7.5 MeV (squares) and $E_{e}=13.4 \mathrm{MeV}$ (circles) at $\gamma$ ELBE. The curves represent the calculated flux described in the text.
TABLE I. Levels assigned to ${ }^{66} \mathrm{Zn}$.

\begin{tabular}{|c|c|c|c|c|}
\hline$E_{x}(\mathrm{keV})^{\mathrm{a}}$ & $I_{\gamma}\left(90^{\circ}\right) / I_{\gamma}\left(127^{\circ}\right)^{\mathrm{b}}$ & $A^{\mathrm{c}}$ & $J_{x}^{\pi \mathrm{d}}$ & $I_{s}(\mathrm{eV} \mathrm{b})^{\mathrm{e}}$ \\
\hline $1040.0(3)^{\mathrm{f}}$ & $1.04(10)$ & & $2^{+}$ & \\
\hline $2779.9(3)^{\mathrm{f}}$ & $1.3(4)$ & & $2^{+}$ & \\
\hline $3380.8(3)$ & $0.92(18)$ & & (1) & $14.4(16)$ \\
\hline $3421.5(3)$ & $1.21(14)$ & & & $4.2(12)$ \\
\hline $3432.1(3)$ & $1.20(6)$ & & & $5.3(14)$ \\
\hline $3737.8(2)$ & $0.82(10)$ & & 1 & $23.7(24)$ \\
\hline $3931.7(3)$ & $1.11(18)$ & & & $12.9(19)$ \\
\hline $4085.7(3)^{\mathrm{g}}$ & $0.7(3)$ & & $1^{+}$ & $6.3(16)$ \\
\hline $4295.0(2)$ & $0.78(11)$ & $+0.97(5)$ & $1^{+}$ & $17.9(21)$ \\
\hline $4426.3(2)$ & $0.71(6)$ & $-0.96(13)$ & $1^{-}$ & $50(4)$ \\
\hline 4461.1(3) & $0.8(3)$ & $+0.80(14)$ & $(1)^{+}$ & $6.2(12)$ \\
\hline $4747.8(3)$ & $0.83(13)$ & $-0.87(27)$ & $1^{-}$ & $20(3)$ \\
\hline $4805.6(2)$ & $0.77(7)$ & $+0.96(5)$ & $1^{+}$ & $62(5)$ \\
\hline 4933.1(6) & $0.85(26)$ & & (1) & $3.1(13)$ \\
\hline $5075.4(2)$ & $0.74(14)$ & $-0.96(8)$ & $1^{-}$ & $35(5)$ \\
\hline $5267.7(3)$ & $0.69(11)$ & $-0.95(10)$ & $1^{-}$ & $21.5(25)$ \\
\hline $5283.0(7)$ & $1.3(4)$ & & & $5.0(14)$ \\
\hline $5329.6(3)$ & $0.76(15)$ & & 1 & $17.5(24)$ \\
\hline $5355.5(3)$ & $0.76(14)$ & $-0.89(17)$ & $1^{-}$ & $15.9(21)$ \\
\hline $5391.0(4)$ & $1.01(22)$ & $-0.82(20)$ & $1^{-}$ & $8.3(20)$ \\
\hline $5399.8(5)$ & $1.8(5)$ & $+0.90(11)$ & $(2)^{+}$ & $10.4(27)$ \\
\hline $5420.1(4)$ & $0.8(4)$ & & (1) & $7.9(25)$ \\
\hline $5439.5(2)$ & $0.80(12)$ & $+0.93(8)$ & $1^{+}$ & $18.3(23)$ \\
\hline $5520.9(2)$ & $0.86(7)$ & $-0.99(5)$ & $1^{-}$ & $52(5)$ \\
\hline $5695.6(2)$ & $0.77(9)$ & $-0.98(6)$ & $1^{-}$ & $32(3)$ \\
\hline $5792.0(3)$ & $0.75(7)$ & $-0.98(8)$ & $1^{-}$ & $22(7)$ \\
\hline $5840.7(1)$ & $0.76(3)$ & $-0.97(2)$ & $1^{-}$ & $222(17)$ \\
\hline $5992.2(2)$ & $0.76(5)$ & $-0.99(3)$ & $1^{-}$ & $82(7)$ \\
\hline $6097.1(2)$ & $0.73(7)$ & $-0.98(3)$ & $1^{-}$ & $47(4)$ \\
\hline $6259.3(3)$ & $0.84(14)$ & $-0.92(12)$ & $1^{-}$ & $19.7(27)$ \\
\hline $6355.3(4)$ & $0.77(14)$ & $<0$ & $1^{-}$ & $19.8(26)$ \\
\hline $6459.3(5)$ & $0.74(23)$ & $-0.45(4)$ & $1^{-}$ & $10.8(19)$ \\
\hline $6533.8(5)$ & $1.07(23)$ & $-0.94(19)$ & $1^{-}$ & $19(3)$ \\
\hline $6571.8(4)$ & $0.84(17)$ & $-0.87(12)$ & $1^{-}$ & $25(4)$ \\
\hline $6691.4(4)$ & $0.76(19)$ & $-0.95(7)$ & $1^{-}$ & $21(4)$ \\
\hline $6720.0(3)$ & $0.76(10)$ & $-0.96(5)$ & $1^{-}$ & $61(6)$ \\
\hline $6830.2(1)$ & $0.65(7)$ & $-0.96(5)$ & $1^{-}$ & 111(10) \\
\hline $6986.6(1)$ & $0.69(6)$ & $-0.95(4)$ & $1^{-}$ & $106(9)$ \\
\hline $7038.5(7)$ & & & & $8(4)$ \\
\hline $7093.1(5)$ & $0.83(17)$ & $-0.96(11)$ & $1^{-}$ & $44(8)$ \\
\hline $7118.7(5)$ & $0.95(15)$ & $<0$ & $1^{-}$ & $50(8)$ \\
\hline $7185.2(3)$ & $0.81(8)$ & $-0.94(3)$ & $1^{-}$ & $70(9)$ \\
\hline $7200.7(3)$ & $0.83(10)$ & $-0.98(10)$ & $1^{-}$ & $53(8)$ \\
\hline $7228.9(5)$ & $0.99(21)$ & $-0.99(23)$ & $1^{-}$ & $26(5)$ \\
\hline $7266.8(2)$ & $0.83(13)$ & $-0.96(5)$ & $1^{-}$ & $69(9)$ \\
\hline $7303.2(4)$ & $0.7(3)$ & $-0.96(6)$ & $1^{-}$ & $33(6)$ \\
\hline $7326.2(2)$ & $0.71(12)$ & $<0$ & $1^{-}$ & $50(8)$ \\
\hline $7367.5(3)$ & $0.89(16)$ & $-0.88(12)$ & $1^{-}$ & $47(7)$ \\
\hline $7393.1(7)$ & $0.90(22)$ & $-0.97(15)$ & $1^{-}$ & $23(5)$ \\
\hline $7413.8(2)$ & $0.87(4)$ & $-0.99(5)$ & $1^{-}$ & $178(22)$ \\
\hline $7446.4(3)$ & $0.71(5)$ & $-0.98(4)$ & $1^{-}$ & $91(12)$ \\
\hline $7486.7(6)$ & $0.90(24)$ & & (1) & 19(4) \\
\hline $7502.6(2)$ & $0.86(10)$ & $-0.96(4)$ & $1^{-}$ & $85(11)$ \\
\hline $7567.3(4)$ & $1.1(4)$ & $-0.95(20)$ & $1^{-}$ & 19(4) \\
\hline $7600.4(3)$ & $0.72(10)$ & $-0.85(10)$ & $1^{-}$ & $56(8)$ \\
\hline $7618.2(4)$ & $0.72(10)$ & & 1 & $48(7)$ \\
\hline $7647.1(3)$ & $0.88(8)$ & $-0.74(11)$ & $1^{-}$ & $102(14)$ \\
\hline 7686.8(4) & $0.50(13)$ & $-0.98(10)$ & $1^{-}$ & $45(9)$ \\
\hline
\end{tabular}


TABLE I. (Continued.)

\begin{tabular}{|c|c|c|c|c|}
\hline$E_{x}(\mathrm{keV})^{\mathrm{a}}$ & $I_{\gamma}\left(90^{\circ}\right) / I_{\gamma}\left(127^{\circ}\right)^{\mathrm{b}}$ & $A^{\mathrm{c}}$ & $J_{x}^{\pi \mathrm{d}}$ & $I_{s}(\mathrm{eV} \mathrm{b})^{\mathrm{e}}$ \\
\hline $7700.1(7)$ & $<0.4$ & $+0.9(5)$ & $\left(1^{+}\right)$ & $25(9)$ \\
\hline $7722.7(3)$ & $0.94(9)$ & $-0.98(6)$ & $1^{-}$ & $70(9)$ \\
\hline $7731.8(3)$ & $0.68(8)$ & $-0.99(3)$ & $1^{-}$ & $77(10)$ \\
\hline $7740.3(4)$ & $0.72(11)$ & $-0.83(17)$ & $1^{-}$ & $44(6)$ \\
\hline $7815.6(3)$ & $0.77(9)$ & $-0.98(7)$ & $1^{-}$ & $57(8)$ \\
\hline $7857.3(8)$ & $0.6(4)$ & $<0$ & $1^{-}$ & $36(12)$ \\
\hline $7900.3(3)$ & $0.91(21)$ & $-0.97(7)$ & $1^{-}$ & $43(7)$ \\
\hline $7912.4(3)$ & $1.1(3)$ & $-0.96(8)$ & $1^{-}$ & $41(7)$ \\
\hline $7933.4(6)$ & $0.69(15)$ & $+0.98(4)$ & $1^{+}$ & $56(11)$ \\
\hline 7958.6(11) & $0.41(12)$ & $<0$ & $1^{-}$ & $30(7)$ \\
\hline $7969.5(6)$ & $0.41(7)$ & $-0.43(20)$ & $1^{-}$ & $67(10)$ \\
\hline $7997.6(8)$ & $0.80(18)$ & $-0.88(10)$ & $1^{-}$ & $38(7)$ \\
\hline $8065.1(4)$ & $0.75(10)$ & $-0.94(9)$ & $1^{-}$ & $77(11)$ \\
\hline $8112.5(3)$ & $0.75(9)$ & $-0.92(7)$ & $1^{-}$ & $76(11)$ \\
\hline $8158.8(5)$ & $1.1(3)$ & $-0.89(11)$ & $1^{-}$ & $53(9)$ \\
\hline $8200.4(5)$ & $0.53(15)$ & $-0.81(13)$ & $1^{-}$ & 61(14) \\
\hline $8266.8(4)$ & $0.87(14)$ & $-0.85(17)$ & $1^{-}$ & $50(8)$ \\
\hline $8349.9(6)$ & $0.87(15)$ & & 1 & $34(5)$ \\
\hline $8366.8(3)$ & $0.70(9)$ & $-0.96(11)$ & $1^{-}$ & $77(11)$ \\
\hline $8457.4(5)$ & $0.81(13)$ & $-0.89(11)$ & $1^{-}$ & $55(8)$ \\
\hline $8476.2(3)$ & $0.80(9)$ & $-0.97(6)$ & $1^{-}$ & $90(12)$ \\
\hline $8530.8(9)$ & $0.52(23)$ & $-0.81(21)$ & $1^{-}$ & $33(11)$ \\
\hline $8597.2(7)$ & $0.93(18)$ & $-0.65(14)$ & $1^{-}$ & $101(21)$ \\
\hline $8688.7(3)$ & $0.64(8)$ & $-0.87(6)$ & $1^{-}$ & $72(10)$ \\
\hline $8714.6(6)$ & $0.47(16)$ & $<0$ & $1^{-}$ & $28(5)$ \\
\hline $8765.7(5)$ & $0.70(11)$ & $-0.97(7)$ & $1^{-}$ & $89(13)$ \\
\hline $8800.2(4)$ & $0.56(13)$ & $-0.79(15)$ & $1^{-}$ & $49(9)$ \\
\hline $8847.3(7)$ & $0.57(19)$ & $-0.96(8)$ & $1^{-}$ & $45(10)$ \\
\hline $8857.8(6)$ & $0.64(18)$ & $+0.99(7)$ & $1^{+}$ & $46(9)$ \\
\hline $8871.1(6)$ & $0.73(22)$ & $-0.6(4)$ & $1^{-}$ & $38(8)$ \\
\hline $8926.5(3)$ & $0.63(6)$ & $-0.93(3)$ & $1^{-}$ & $92(12)$ \\
\hline $8987.0(5)$ & $0.9(6)$ & $-0.89(17)$ & $1^{-}$ & $51(15)$ \\
\hline $9009.7(4)$ & $0.59(14)$ & $-0.88(15)$ & $1^{-}$ & $61(11)$ \\
\hline $9044.2(5)$ & $0.47(12)$ & $+0.7(3)$ & $1^{+}$ & $40(8)$ \\
\hline $9102.4(3)$ & $0.73(9)$ & $-0.98(15)$ & $1^{-}$ & $79(11)$ \\
\hline $9210.0(8)$ & $0.53(17)$ & $-0.99(4)$ & $1^{-}$ & $104(28)$ \\
\hline $9293.0(7)$ & $0.58(16)$ & & 1 & $38(8)$ \\
\hline $9317.4(6)$ & $0.49(10)$ & $-0.94(9)$ & $1^{-}$ & $54(9)$ \\
\hline $9349.0(5)$ & $0.63(8)$ & $-0.99(4)$ & $1^{-}$ & $74(11)$ \\
\hline $9456.7(6)^{\mathrm{h}}$ & $0.71(14)$ & & 1 & $31(5)$ \\
\hline $9470.9(4)$ & $0.74(9)$ & $-0.92(7)$ & $1^{-}$ & $53(8)$ \\
\hline 9491.1(5) & $0.80(11)$ & $-0.52(24)$ & $1^{-}$ & $47(7)$ \\
\hline $9504.5(5)$ & $0.63(11)$ & & 1 & $39(6)$ \\
\hline $9521.2(6)$ & $0.71(14)$ & $-0.96(9)$ & $1^{-}$ & $36(6)$ \\
\hline $9541.8(3)$ & $0.57(7)$ & $-0.92(23)$ & $1^{-}$ & $78(11)$ \\
\hline $9578.5(5)$ & $1.3(6)$ & $-0.89(19)$ & $1^{-}$ & $40(8)$ \\
\hline $9648.9(5)$ & $0.90(14)$ & $-0.91(7)$ & $1^{-}$ & $58(9)$ \\
\hline $9683.4(4)$ & $0.86(11)$ & $-0.93(6)$ & $1^{-}$ & $80(11)$ \\
\hline $9726.2(6)$ & $0.42(11)$ & $-0.98(15)$ & $1^{-}$ & $53(10)$ \\
\hline $9743.8(6)$ & $0.89(27)$ & $-0.91(13)$ & $1^{-}$ & $47(9)$ \\
\hline 9763.9(12) & & & & $22(7)$ \\
\hline $9802.5(4)$ & $0.63(10)$ & $-0.79(19)$ & $1^{-}$ & $108(17)$ \\
\hline $9824.9(6)$ & $0.41(17)$ & & & $36(10)$ \\
\hline $9850.3(8)$ & $0.50(16)$ & $<0$ & $1^{-}$ & $65(19)$ \\
\hline $9869.8(12)$ & $0.42(9)$ & & 1 & $35(11)$ \\
\hline $9949.2(5)$ & $0.64(17)$ & $-0.90(7)$ & $1^{-}$ & $45(9)$ \\
\hline $9968.0(4)$ & $0.81(15)$ & $-0.98(18)$ & $1^{-}$ & $60(10)$ \\
\hline $9992.4(5)$ & $0.7(3)$ & $-0.95(8)$ & $1^{-}$ & $62(15)$ \\
\hline
\end{tabular}

TABLE I. (Continued.)

\begin{tabular}{lclll}
\hline \hline$E_{x}(\mathrm{keV})^{\mathrm{a}}$ & $I_{\gamma}\left(90^{\circ}\right) / I_{\gamma}\left(127^{\circ}\right)^{\mathrm{b}}$ & \multicolumn{1}{c}{$A^{\mathrm{c}}$} & $J_{x}^{\pi \mathrm{d}}$ & $I_{s}(\mathrm{eV} \mathrm{b})^{\mathrm{e}}$ \\
\hline $10016.8(6)$ & $0.69(27)$ & $-0.94(7)$ & $1^{-}$ & $58(14)$ \\
$10054.1(6)$ & $0.61(13)$ & $-0.76(14)$ & $1^{-}$ & $76(13)$ \\
$10109.8(9)$ & $0.6(4)$ & & 1 & $53(19)$ \\
$10126.0(11)$ & $0.7(6)$ & & $(1)$ & $65(29)$ \\
$10145.2(8)$ & $0.6(3)$ & & 1 & $29(11)$ \\
$10196.2(10)$ & $0.7(5)$ & & $(1)$ & $37(16)$ \\
$10230.7(7)$ & & & $16(6)$ \\
$10243.8(6)$ & $0.7(4)$ & & $(1)$ & $18(6)$ \\
$10433.7(9)$ & $0.63(17)$ & & 1 & $48(11)$ \\
$10530.0(7)$ & $0.61(18)$ & & 1 & $34(8)$ \\
$10843.2(7)$ & $0.48(19)$ & & 1 & $14(3)$ \\
$10858.6(5)$ & $0.76(18)$ & & 1 & $21(4)$ \\
$10937.7(5)$ & $0.50(13)$ & & 1 & $58(12)$ \\
$10991.0(7)$ & $1.3(7)$ & & $20(8)$ \\
$11015.8(10)$ & $0.5(3)$ & & 1 & $30(11)$ \\
\hline \hline
\end{tabular}

${ }^{a}$ Excitation energy. The uncertainty of this and the other quantities in the table is given in parentheses in units of the last digit. This energy value was deduced from the $\gamma$-ray energy measured at $127^{\circ}$ including a recoil and Doppler-shift correction.

${ }^{b}$ Ratio of the intensities measured at angles of $90^{\circ}$ and $127^{\circ}$. The expected values for an elastic dipole transition (spin sequence 0-1-0) and for an elastic quadrupole transition (spin sequence 0-2-0) are 0.74 and 2.15 , respectively.

${ }^{\mathrm{c}}$ Azimuthal asymmetry $A=\left(I_{\gamma \mathrm{H}}-I_{\gamma \mathrm{V}}\right) /\left(I_{\gamma \mathrm{H}}+I_{\gamma \mathrm{V}}\right)$ of the intensities $I_{\gamma \mathrm{H}}$ and $I_{\gamma \mathrm{V}}$ measured with the detectors placed in a horizontal and vertical plane, respectively. A negative asymmetry indicates $E 1$ radiation and a positive asymmetry indicates $M 1 / E 2$ radiation.

${ }^{\mathrm{d}}$ Spin and parity deduced from angular correlation and azimuthal asymmetry, respectively, of the ground-state transition.

${ }^{\text {e}}$ Energy-integrated scattering cross section. Below an excitation energy of $7.0 \mathrm{MeV}$ the value was deduced from the measurement at 7.5 MeV electron energy, otherwise the value was deduced from the measurement at $13.4 \mathrm{MeV}$.

${ }^{\mathrm{f}}$ This state is assumed to be a known $2^{+}$state [67].

${ }^{\mathrm{g}}$ This state is assumed to be a known $1^{+}$state [67].

${ }^{\mathrm{h}} \mathrm{Up}$ to about $40 \%$ of the intensity of the ground-state transition may belong to a single-escape peak.

work [47] in Table II. One sees that the feeding intensities decrease toward high energy and become negligible above about $5 \mathrm{MeV}$, which corresponds to the findings of previous studies $[68,69]$. The transitions considered as inelastic transitions are listed in Table III together with the corresponding ground-state transitions and the ratios of their intensities.

\section{E. Experiments with monoenergetic and linearly polarized photon beams at $\mathrm{HI} \gamma \mathrm{S}$}

Monoenergetic photon beams are produced at $\mathrm{HI} \gamma \mathrm{S}$ by Compton back-scattering of a high-intensity free-electron laser (FEL) beam from an electron beam in the TUNL storage ring. Presently, the energy of the backward scattered photons can be tuned over a wide energy range, from about 1 to $100 \mathrm{MeV}$, by changing the energy of the electron beam and the FEL wavelength [27]. The polarization of the FEL photons, defined by the magnetic field of the undulators, is mostly 
TABLE II. Comparison of level widths and spin and parity assignments deduced from the present measurements with those from previous work.

\begin{tabular}{lccccc}
\hline \hline $\begin{array}{l}E_{x}{ }^{\mathrm{a}} \\
(\mathrm{keV})\end{array}$ & $\begin{array}{c}\Gamma_{0}^{2} / \Gamma^{\mathrm{b}} \\
(\mathrm{meV}) \\
13.4 \mathrm{MeV}\end{array}$ & $\begin{array}{c}\Gamma_{0}^{2} / \Gamma^{\mathrm{c}} \\
(\mathrm{meV}) \\
7.5 \mathrm{MeV}\end{array}$ & $\begin{array}{c}\Gamma_{0}^{2} / \Gamma^{\mathrm{d}} \\
(\mathrm{meV}) \\
\text { Previous }\end{array}$ & $\begin{array}{c}J^{\pi} \\
\text { Present }\end{array}$ & $\begin{array}{c}J^{\pi \mathrm{e}} \\
\text { Previous }\end{array}$ \\
\hline 3381 & $91(11)$ & $14.3(16)$ & $11.0(21)$ & $(1)$ & $1^{(+)}$ \\
3432 & $39(5)$ & $5.4(14)$ & $4.1(15)$ & & $\left(1^{-}\right)$ \\
3738 & $81(10)$ & $28.7(29)$ & $24(3)$ & 1 & $1^{(-)}$ \\
4295 & $63(8)$ & $29(3)$ & $40(12)$ & $1^{+}$ & $1^{(+)}$ \\
4426 & $96(12)$ & $85(7)$ & $65(10)$ & $1^{-}$ & $1^{(-)}$ \\
4461 & $30(4)$ & $10.7(21)$ & $8(6)$ & $(1)^{+}$ & $1^{(+)}$ \\
4806 & $120(15)$ & $124(10)$ & $81(20)$ & $1^{+}$ & $1^{+}$ \\
\hline \hline
\end{tabular}

${ }^{\mathrm{a}}$ Level energy.

${ }^{\mathrm{b}}$ Quantity deduced from the integrated scattering cross section determined from the measurement at $13.4 \mathrm{MeV}$ electron energy according to Eq. (2).

${ }^{\mathrm{C}}$ Quantity deduced from the integrated scattering cross section determined from the measurement at $7.5 \mathrm{MeV}$ electron energy (see Table I) according to Eq. (2).

${ }^{\mathrm{d}}$ Widths of the ground-state transitions $\Gamma_{0}$ taken from Ref. [47]. Branching ratios $\Gamma_{0} / \Gamma$ of the ground-state transitions taken from Ref. [48] for the states at 3381, 3432, 4295, 4461, $4806 \mathrm{keV}$, and assumed to be one for the 3738 and $4426 \mathrm{keV}$ states.

${ }^{\mathrm{e}}$ Spins taken from Ref. [47]. Parities taken from Refs. [48-50].

preserved during the Compton backscattering process due to a negligible recoil effect, leading to the production of intense photon beams with nearly $100 \%$ polarization.

The measurements at $\mathrm{HI} \gamma \mathrm{S}$ were carried out at photonbeam energies of $4.3,4.4,4.55,4.7,4.85,5.0,5.15,5.3,5.45$, 5.6, 5.75, 5.9, 6.1, 6.3, 6.5, 6.7, 6.9, 7.1, 7.3, 7.5, 7.7, 7.9, 8.15, $8.4,8.65,8.9,9.15,9.4,9.65$, and $9.9 \mathrm{MeV}$. The energy spread (FWHM) of the beam was about $3 \%$ of the energy using a $30.5-\mathrm{cm}$-long lead collimator with a cylindrical hole of $1.9 \mathrm{~cm}$ diameter positioned $60 \mathrm{~m}$ downstream from the collision point of the electrons with the FEL photons. The measuring time was on average about $3 \mathrm{~h}$ for each selected energy. The photon beam impinged onto the target with a flux of about $5 \times 10^{6} \mathrm{~s}^{-1}$ for the lowest energies and up to $3 \times 10^{7} \mathrm{~s}^{-1}$ for the highest energies. Scattered photons were measured with two HPGe detectors placed at polar angles of $90^{\circ}$ to the beam. One

TABLE III. Assumed inelastic transitions feeding the $2_{1}^{+}$state.

\begin{tabular}{lll}
\hline \hline$E_{\gamma 0}(\mathrm{keV})^{\mathrm{a}}$ & $E_{\gamma 1}(\mathrm{keV})^{\mathrm{b}}$ & $I_{\gamma 1} / I_{\gamma 0} \mathrm{c}$ \\
\hline $5075.0(2)$ & $4035.8(3)$ & $0.39(8)$ \\
$5695.1(2)$ & $4655.8(5)$ & $0.40(7)$ \\
$5991.5(2)$ & $4952.2(4)$ & $0.05(2)$ \\
$6571.1(4)$ & $5530.6(5)$ & $0.35(10)$ \\
$6719.2(3)$ & $5680.5(4)$ & $0.21(5)$ \\
$8845.9(7)$ & $7805.5(9)$ & $0.42(11)$ \\
$10015.0(6)$ & $8976.5(11)$ & $0.7(3)$ \\
\hline \hline
\end{tabular}

${ }^{\mathrm{a}}$ Energy of the elastic transition to the ground state.

${ }^{\mathrm{b}}$ Energy of the inelastic transition to the $2_{1}^{+}$state.

${ }^{\mathrm{c}}$ Ratio of the intensities of the inelastic and elastic transitions.

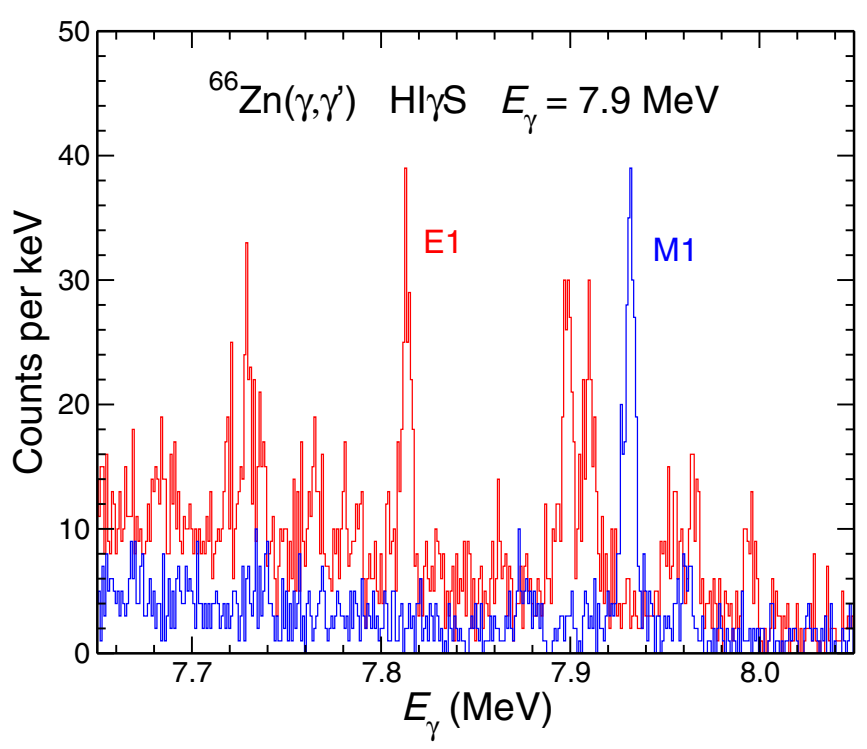

FIG. 3. Parts of spectra of photons scattered from ${ }^{66} \mathrm{Zn}$, measured during the irradiation with a quasimonoenergetic polarized photon beam of $7.9 \mathrm{MeV}$ at $\mathrm{HI} \gamma \mathrm{S}$. The spectrum plotted in red was measured with the vertical detector and contains $E 1$ radiation, whereas the spectrum plotted in blue was measured with the horizontal detector and contains $M 1$ radiation.

detector of $80 \%$ relative efficiency was positioned vertical and one of $100 \%$ relative efficiency horizontal to the beam tube. The type of radiation ( $E 1$ or $M 1)$ was deduced from a comparison of the intensities of the transitions measured at the different azimuthal angles relative to the polarization plane $[53,70]$. In the present setup, $E 1$ radiation is detected preferentially in the vertical detector and $M 1$ radiation in the horizontal one. As an example, spectra measured at a beam energy of $7.9 \mathrm{MeV}$ are given in Fig. 3. The spectra allow a clear distinction between $E 1$ and $M 1$ radiation. Azimuthal asymmetries $A=\left(I_{\gamma \mathrm{H}}-I_{\gamma \mathrm{V}}\right) /\left(I_{\gamma \mathrm{H}}+I_{\gamma \mathrm{V}}\right)$ deduced from the intensities $I_{\gamma \mathrm{H}}$ measured in the horizontal detector and $I_{\gamma \mathrm{V}}$ measured in the vertical one are given in Table I together with the resulting parities assigned to the emitting states.

\section{DETERMINATION OF THE DIPOLE-STRENGTH DISTRIBUTION}

The determination of the dipole-strength distribution and the related photoabsorption cross section requires the knowledge of the intensity distribution of the ground-state transitions and their branching ratios. As these cannot be derived directly from the measured spectra, we applied statistical methods to discriminate between $\gamma$ rays from nuclear excitations and photons scattered by atomic processes, and to disentangle the intensity distributions of elastic and inelastic transitions in the quasicontinuum of nuclear levels.

First, a spectrum of the ambient background adjusted to the intensities of the transitions from ${ }^{40} \mathrm{~K}$ and ${ }^{208} \mathrm{Tl}$ decay in the in-beam spectrum was subtracted from the measured spectrum. To correct the measured spectrum for the detector response, spectra of monoenergetic $\gamma$ rays were calculated in 


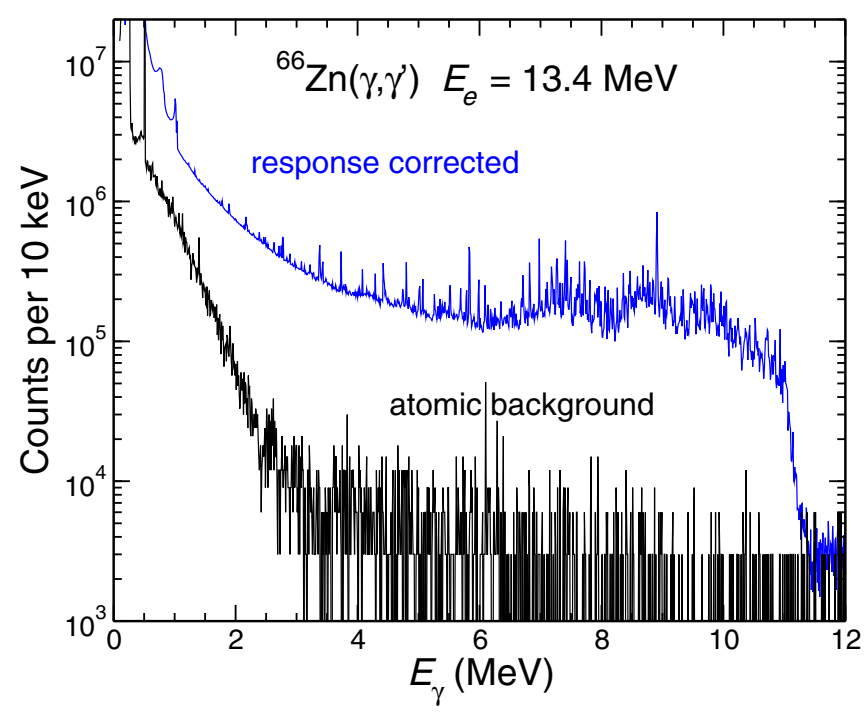

FIG. 4. Spectrum of the two detectors at $127^{\circ}$, corrected for detector response (blue), and simulated spectrum of photons scattered from the target to the detectors by atomic processes (black).

steps of $10 \mathrm{keV}$ by using the simulation code GEANT4. Starting from the high-energy end of the experimental spectrum, the simulated spectra were subtracted sequentially (spectrumstripping method) [71].

The background radiation produced by atomic processes in the ${ }^{66} \mathrm{Zn}$ target was obtained from a GEANT4 simulation. The simulated atomic background is compared with the responsecorrected spectrum in Fig. 4. The atomic background amounts in average to only a few percent of the intensity in the spectrum, but coincides with that above the neutron threshold, which proves the right magnitude. This behavior is similar to that found in previous studies $[28-30,32,33,37,59,72]$ and shows that the experimental spectrum contains a considerable amount of nuclear strength in a quasicontinuum. This is formed by a large number of unresolved transitions with small intensities that are the result of the increasing nuclear level density at high energy in combination with the finite detector resolution. Because of the different orders of magnitude, the nuclear intensity distribution resulting from the subtraction of the simulated atomic background is not very sensitive to uncertainties of the latter, for which we assume $5 \%$. The nuclear intensity distribution contains ground-state (elastic) transitions and, in addition, branching (inelastic) transitions to lower-lying excited states as well as transitions from those states to the ground state (cascade transitions). The different types of transitions cannot be clearly distinguished. However, for the determination of the photoabsorption cross section and the partial widths $\Gamma_{0}$, the intensities of the ground-state transitions are needed. Therefore, contributions of inelastic and cascade transitions have to be subtracted from the spectra. We corrected the intensity distributions by simulating $\gamma$-ray cascades from the levels in the entire energy region using the code $\gamma$ DEX $[60,73]$. This code works analogously to the strategy of the code DICEBOX [74] developed for $(n, \gamma)$ reactions, but in addition it includes also the excitation from the ground state. In the present simulations, level schemes (nuclear realiza- tions) including states with $J=0, \ldots, 5$ were created. Known low-lying levels were taken into account up to about 3.5 $\mathrm{MeV}$. Partial widths were varied in the individual nuclear realizations applying the Porter-Thomas distribution [75]. Level densities were calculated by using the constant-temperature model [76] with the parameters $T=1.42$ (3) $\mathrm{MeV}$ and $E_{0}=$ $0.04(11) \mathrm{MeV}$ adjusted to experimental level densities [77]. In the individual nuclear realizations, the values of $T$ and $E_{0}$ were varied randomly within a Gaussian distribution with a $\sigma$ corresponding to the uncertainties given in Ref. [77]. The parity distribution of the level densities was modeled according to the information given in Ref. [78].

The first input for the photon strength function simulations were assumed to be Lorentz-shaped. For the $E 1$ strength, a sum of three Lorentz functions (TLO) that account for a triaxial deformation of the nucleus was used with parameters described in Refs. [79,80]. In the present case, deformation parameters of $\beta_{2}=0.22$ [81] and $\gamma=29^{\circ}$ [82] were applied. The parameters for the $M 1$ and $E 2$ strengths were taken from global parametrizations of $M 1$ spin-flip resonances and $E 2$ isoscalar resonances, respectively [83]. Spectra of $\gamma$-ray cascades were generated for groups of levels in energy bins of $\Delta E=100 \mathrm{keV}$. Starting from the high-energy end of the intensity distribution, that contains ground-state transitions only, the simulated intensities of the ground-state transitions were normalized to the experimental ones in the considered bin. The intensity distribution of the branching transitions was subtracted from the total intensity distribution. Applying this procedure step by step for each energy bin moving toward the low-energy end of the spectrum, one obtains the intensity distribution of the ground-state transitions. Simultaneously, the branching ratios $b_{0}(E)$ of the ground-state transitions are deduced for each energy bin. In an individual nuclear realization, the branching ratio $b_{0}(E)$ is calculated as the ratio of the sum of the intensities of the ground-state transitions from all levels in $\Delta E$ to the total intensity of all transitions depopulating those levels to either any low-lying levels and the ground state $[10,29,30,32,60,73]$. Branching ratios $\left\langle b_{0}(E)\right\rangle$, averaged over the many nuclear realizations from the present cascade simulations, are illustrated in Fig. 5.

The uncertainty of the number of counts $N(E)$ in an energy bin of the experimental intensity distribution was deduced as

$$
\delta N(E)=\sqrt{N(E)}+\sum_{E^{\prime}}\left[\sqrt{N\left(E^{\prime}>E\right)} b\left(E^{\prime} \rightarrow E\right)\right],
$$

where $b\left(E^{\prime} \rightarrow E\right)$ is the branching intensity from bin $E^{\prime}$ to bin $E$. We transform $N(E)$ to the scattering cross section according to

$$
\sigma_{\gamma \gamma}(E)=N(E) /\left[\epsilon(E) \Phi_{\gamma}(E) W(E) N_{N} \Delta t \Delta E\right]
$$

with the quantities defined in Eq. (1), the absolute detector efficiency $\epsilon(E)$, the measuring time $\Delta t$, and the bin width $\Delta E$. The absorption cross section in each bin is obtained as $\sigma_{\gamma}(E)=\sigma_{\gamma \gamma}(E) / b_{0}(E)$ for each nuclear realization. Finally, the absorption cross sections of each bin were obtained by averaging over the values of the individual nuclear realizations.

The simulations were performed iteratively, where the strength function obtained from an iteration step was used as the input for the next step. We note that the simulations are 


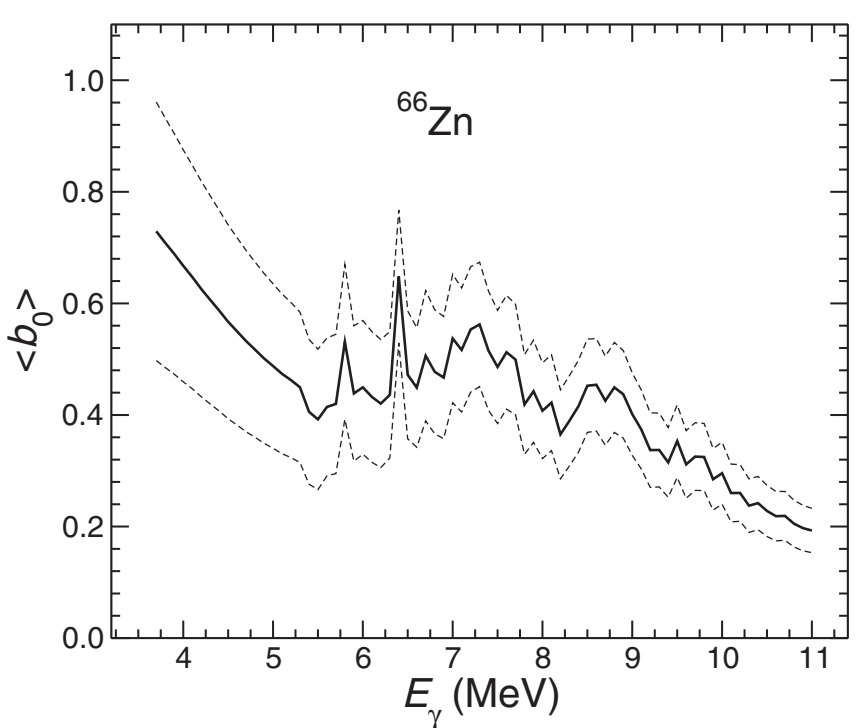

FIG. 5. Average branching ratios (solid curve) with their uncertainty ranges (dashed curves) resulting from the simulations of statistical $\gamma$-ray cascades up to $S_{n}$ as described in the text.

little sensitive to the shape of the first input strength function, which was tested, for example, in Refs. [68,73]. The iteration was stopped when the input strength function and the output strength function were in agreement within their respective uncertainties, which was achieved in the fourth iteration step. The cross section obtained in the last iteration step is adopted as the final absorption cross section. The uncertainties of the cross-section values include statistical uncertainties of the spectrum, the given uncertainties of the efficiency and the subtracted simulated background spectra, uncertainties of the flux resulting from the integrated cross sections of the ${ }^{11} \mathrm{~B}$ levels, and the given uncertainties of the level-density parameters. Toward low energy, the uncertainties increase due to the use of the spectrum-stripping method and the cross sections do not converge. Therefore, cross sections cannot be determined below an excitation energy of $5.4 \mathrm{MeV}$ in the high-energy measurement. The absorption cross section obtained from the procedure just described is listed in Table IV and graphed in Fig. 6.

To obtain values toward lower energies, we applied the same procedure to the low-energy measurement. However, this procedure starts at comparably small energies, at which the level density is small and the statistical method is at the limits of its applicability. For comparison, the result is also shown in Fig. 6. The cross section values are uncertain and fluctuate strongly. In particular, there are two steep decreases with a fluctuation to higher values in between. Besides, the values do not continue much further to low energies compared with the values from the high-energy measurement. This demonstrates the limits of the described methods and is not considered reliable for applications.
TABLE IV. Photoabsorption cross section deduced from the present $\left(\gamma, \gamma^{\prime}\right)$ experiment at $E_{e}=13.4 \mathrm{MeV}$.

\begin{tabular}{|c|c|}
\hline$E_{\gamma}(\mathrm{MeV})$ & $\sigma(\mathrm{mb})^{\mathrm{a}}$ \\
\hline 5.4 & $0.92(22)$ \\
\hline 5.5 & $1.4(4)$ \\
\hline 5.7 & $0.94(19)$ \\
\hline 5.8 & $0.7(5)$ \\
\hline 6.0 & $0.68(12)$ \\
\hline 6.1 & $0.90(14)$ \\
\hline 6.2 & $1.02(16)$ \\
\hline 6.3 & $0.99(14)$ \\
\hline 6.5 & $1.6(4)$ \\
\hline 6.6 & $1.5(3)$ \\
\hline 6.7 & $2.1(10)$ \\
\hline 6.8 & $2.6(13)$ \\
\hline 6.9 & $2.4(9)$ \\
\hline 7.0 & $3.0(19)$ \\
\hline 7.1 & $3.1(16)$ \\
\hline 7.2 & $3.8(23)$ \\
\hline 7.3 & $4.4(27)$ \\
\hline 7.4 & $6(3)$ \\
\hline 7.5 & $4.5(18)$ \\
\hline 7.6 & $5.6(27)$ \\
\hline 7.7 & $6.0(26)$ \\
\hline 7.8 & $6.1(20)$ \\
\hline 7.9 & $6.8(23)$ \\
\hline 8.0 & $6.4(15)$ \\
\hline 8.1 & $7.2(16)$ \\
\hline 8.2 & 7.1(11) \\
\hline 8.3 & $7.7(13)$ \\
\hline 8.4 & $9.3(23)$ \\
\hline 8.5 & $10.0(26)$ \\
\hline 8.6 & 11(3) \\
\hline 8.7 & $11.4(28)$ \\
\hline 8.8 & $12(3)$ \\
\hline 8.9 & $11.7(28)$ \\
\hline 9.0 & $11.3(20)$ \\
\hline 9.1 & $12.7(22)$ \\
\hline 9.2 & $10.4(6)$ \\
\hline 9.3 & 11.6(9) \\
\hline 9.4 & $10.3(7)$ \\
\hline 9.5 & 14.1(7) \\
\hline 9.6 & $13.0(4)$ \\
\hline 9.7 & $14.5(6)$ \\
\hline 9.8 & $14.5(4)$ \\
\hline 9.9 & $13.3(5)$ \\
\hline 10.0 & $14.4(5)$ \\
\hline 10.1 & $13.3(6)$ \\
\hline 10.2 & $12.5(8)$ \\
\hline 10.3 & $11.4(10)$ \\
\hline 10.4 & $12.8(10)$ \\
\hline 10.5 & $12.0(11)$ \\
\hline 10.6 & 11.1(12) \\
\hline 10.7 & $12.5(12)$ \\
\hline 10.8 & $10.3(13)$ \\
\hline 10.9 & $10.0(14)$ \\
\hline
\end{tabular}


TABLE IV. (Continued.)

\begin{tabular}{ll}
\hline \hline$E_{\gamma}(\mathrm{MeV})$ & $\sigma(\mathrm{mb})^{\mathrm{a}}$ \\
\hline 11.0 & $8.8(14)$ \\
11.1 & $7.0(21)$ \\
\hline \hline
\end{tabular}

$\overline{{ }^{a} \text { Absorption cross section resulting from the experimental intensity }}$ distribution including the quasicontinuum, corrected for branching intensities and branching ratios obtained from $\gamma$-ray cascade simulations. The uncertainties include statistical uncertainties of the included intensities as described in Sec. III, 5\% uncertainties of the detector efficiencies and the simulated atomic background, and uncertainties of the photon flux based on the uncertainties of the cross sections in ${ }^{11}$ B. Systematic uncertainties of level-density models can result in additional uncertainties of up to about $20 \%$, which are not included here.

Scattering and absorption cross sections can also be deduced from the experiments with quasimonoenergetic photon beams at $\mathrm{HI} \gamma \mathrm{S}$. The advantage is that only a bunch of states in an energy window of typically $200 \mathrm{keV}$ is excited. These states are not affected by feeding and the corresponding inelastic transitions can clearly be identified, if they are intense enough. This allows in principle the determination of the cross sections without the application of model-dependent cascade simulations. There may be, however, also weak unresolved transitions in the spectrum, which requires a treatment of the quasicontinuum as well. The disadvantage of these experiments is the necessary unfolding of the detected energy distribution of the incident photon beam based on simulations, and the need of additional data for the calibration of the photon flux, which is usually performed on the basis of cross sections of individual transitions known from earlier

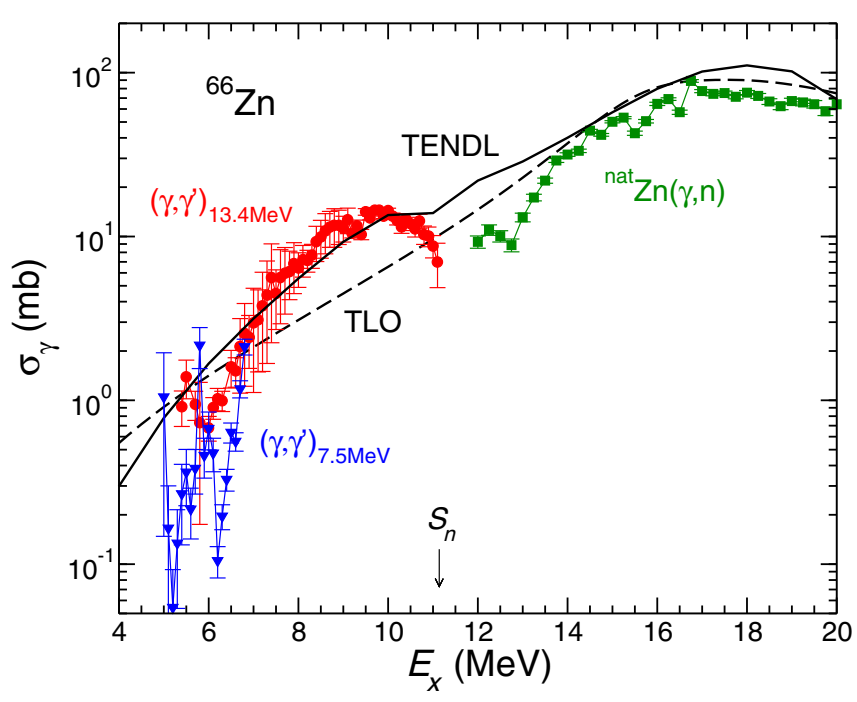

FIG. 6. Photoabsorption cross sections of ${ }^{66} \mathrm{Zn}$ resulting from the present $\left(\gamma, \gamma^{\prime}\right)$ experiments at $E_{e}=7.5 \mathrm{MeV}$ (blue triangles) and $E_{e}=13.4 \mathrm{MeV}$ (red circles), from $(\gamma, n)$ data for ${ }^{\text {nat }} \mathrm{Zn}$ (green squares) taken from Ref. [86], from calculations using the TALYS code as given in the TENDL-2019 library (black solid curve), and from the TLO with parameters given in the text (black dashed curve).

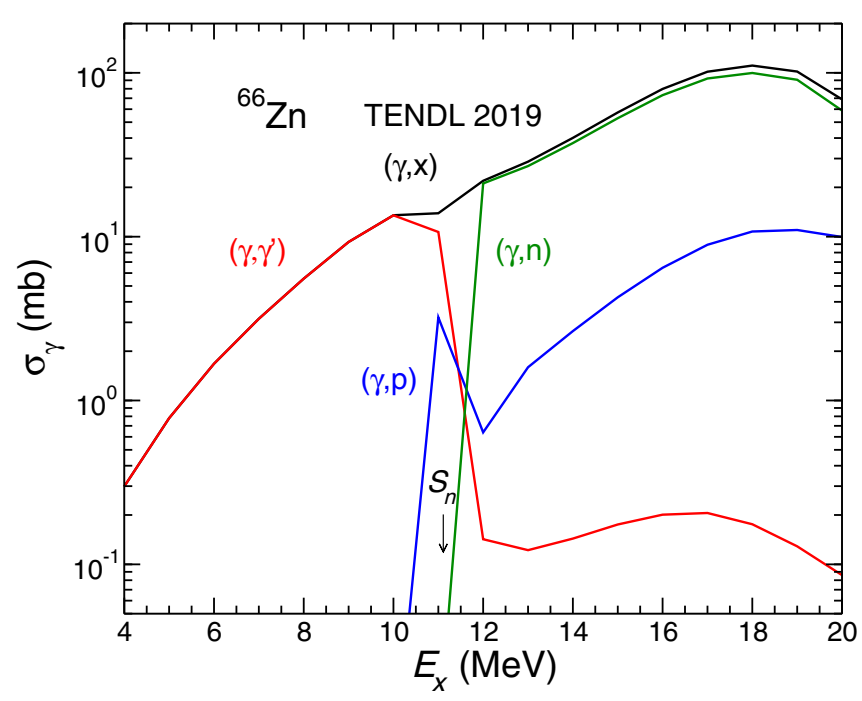

FIG. 7. Photoabsorption cross section of ${ }^{66} \mathrm{Zn}$ (black curve) together with the partial cross sections for the reaction channels $\left(\gamma, \gamma^{\prime}\right)$ (red curve), $(\gamma, n)$ (green curve), and $(\gamma, p)$ (blue curve), taken from the TENDL-2019 library.

experiments with bremsstrahlung. In previous experiments, we compared average branching ratios $\left\langle b_{0}(E)\right\rangle$ from experiments at both $\gamma$ ELBE and $\mathrm{HI} \gamma \mathrm{S}$, and found a good agreement, for example for the nuclides ${ }^{136} \mathrm{Ba}$ [60] and ${ }^{128,134} \mathrm{Xe}$ [37]. Moreover, the cross section in ${ }^{136} \mathrm{Ba}$ [60] deduced from experiments at $\gamma \mathrm{ELBE}$ is consistent with that in the neighbor ${ }^{138} \mathrm{Ba}$ obtained at $\mathrm{HI} \gamma \mathrm{S}$ [84]. A good agreement of the cross sections deduced from the described analysis of $\gamma \mathrm{ELBE}$ data with those deduced from $\mathrm{HI} \gamma \mathrm{S}$ data was also achieved in a recent study of ${ }^{87} \mathrm{Rb}$ [85]. We plan the determination of absorption cross sections in ${ }^{66} \mathrm{Zn}$ also on the basis of the present $\mathrm{HI} \gamma \mathrm{S}$ data. For this purpose, the integrated cross sections in Table I can be used to deduce the photon flux in the selected beam-energy windows. This complex analysis is the subject of a forthcoming paper.

\section{DISCUSSION}

The photoabsorption cross section resulting from the present experiments is compared with the cross section for the $(\gamma, n)$ reaction [86] in Fig. 6. In addition, the TLO with the parameters just mentioned and the photoabsorption cross section given in the latest TALYS-based evaluated nuclear data library (TENDL-2019) [87] are displayed as well. Therein, the standard Lorentzian (Brink-Axel model) $[2,13]$ was used for the $\left(\gamma, \gamma^{\prime}\right)$ reaction [88]. The TENDL cross section is composed of the cross sections of various reaction channels shown in Fig. 7. The present $\left(\gamma, \gamma^{\prime}\right)$ cross section shows pronounced extra strength above the TLO in the energy region from about $6 \mathrm{MeV}$ to $S_{n}$, which is attributed to the PDR. The experimental cross section is well approximated by the TENDL cross section because of its smooth behavior. This differs considerably from the strongly fluctuating cross section of the $N=28$ nuclide ${ }^{54} \mathrm{Fe}$ [46], which is compared with the one of ${ }^{66} \mathrm{Zn}$ in Fig. 8. Those fluctuations in ${ }^{54} \mathrm{Fe}$ are caused by prominent excitations manifesting in intense isolated peaks 


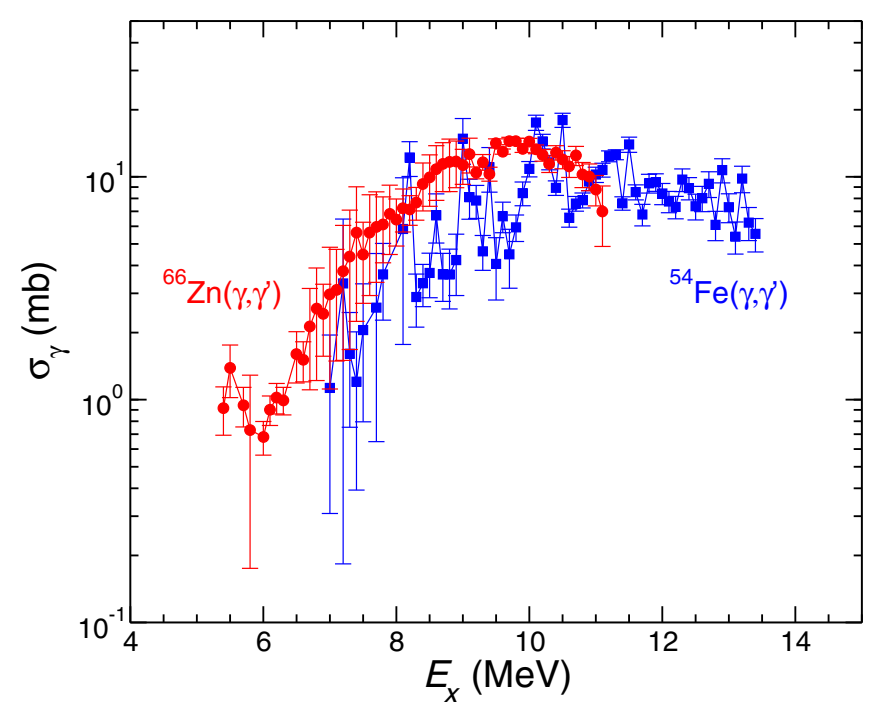

FIG. 8. Photoabsorption cross sections of ${ }^{66} \mathrm{Zn}$ from the present experiments (red circles) and of ${ }^{54} \mathrm{Fe}$ taken from Ref. [46] (blue squares).

in the spectrum of ${ }^{54} \mathrm{Fe}$. In ${ }^{66} \mathrm{Zn}$, there are fewer such intense peaks but a greater number of peaks with average intensity. There are 128 peaks between 3.4 and $11.0 \mathrm{MeV}$ in ${ }^{66} \mathrm{Zn}$ (cf. Table I) compared to about 100 between 5.1 and $13.2 \mathrm{MeV}$ in ${ }^{54} \mathrm{Fe}$. The higher level density combined with fewer prominent peaks in the open-shell nuclide ${ }^{66} \mathrm{Zn}$ leads to a smoother curve for the photoabsorption cross section compared to that in the $N=28$ nuclide ${ }^{54} \mathrm{Fe}$.

Photon strength functions can also be determined in lightion induced reactions, applying the so-called Oslo method [89]. While the strength functions deduced from $\left(\gamma, \gamma^{\prime}\right)$ data contain exclusively ground-state transitions from $J=1$ states, the strength functions obtained from light-ion induced reactions comprise a large number of transitions linking many states of various spins up to about $J=10$, which may cause different characteristics of the strength functions. The nearest neighbor of ${ }^{66} \mathrm{Zn}$, for which such data are available, is ${ }^{64} \mathrm{Ni}$. In Fig. 9, the photon strength function $f$ deduced from the present cross section data for ${ }^{66} \mathrm{Zn}$ is compared with the one obtained from $\left(p, p^{\prime} \gamma\right)$ experiments for ${ }^{64} \mathrm{Ni}[90]$. The $f$ of ${ }^{66} \mathrm{Zn}$ crosses the one of ${ }^{64} \mathrm{Ni}$ in the range from about 6 to $7 \mathrm{MeV}$ and is nearly parallel, but greater by about $50 \%$ at higher energies, still lying inside the extreme limits of the $f$ of ${ }^{64} \mathrm{Ni}$. The latter continues toward small $\gamma$-ray energies, including transitions between close-lying states at high excitation energy, and displays the beginning upbend below about 3.5 $\mathrm{MeV}$, which cannot be identified from conventional singles $\left(\gamma, \gamma^{\prime}\right)$ data (see Sec. I and Ref. [25]).

The relation between $E 1$ and $M 1$ strengths in ${ }^{66} \mathrm{Zn}$ can be analyzed only for the intense transitions from states with parity assignments given in Table I. The summed integrated scattering cross section of all definite and tentative $1^{-}$states in Table I amounts to $\sum I_{s}\left(1^{-}\right)=5019(94) \mathrm{eV} \mathrm{b}$ and that of $1^{+}$ states amounts to $\sum I_{S}\left(1^{+}\right)=278(20) \mathrm{eV}$ b. The contribution of $M 1$ strength is hence about $5 \%$ of the total dipole strength from the resolved states. Compared to the $45 \%$ contribution

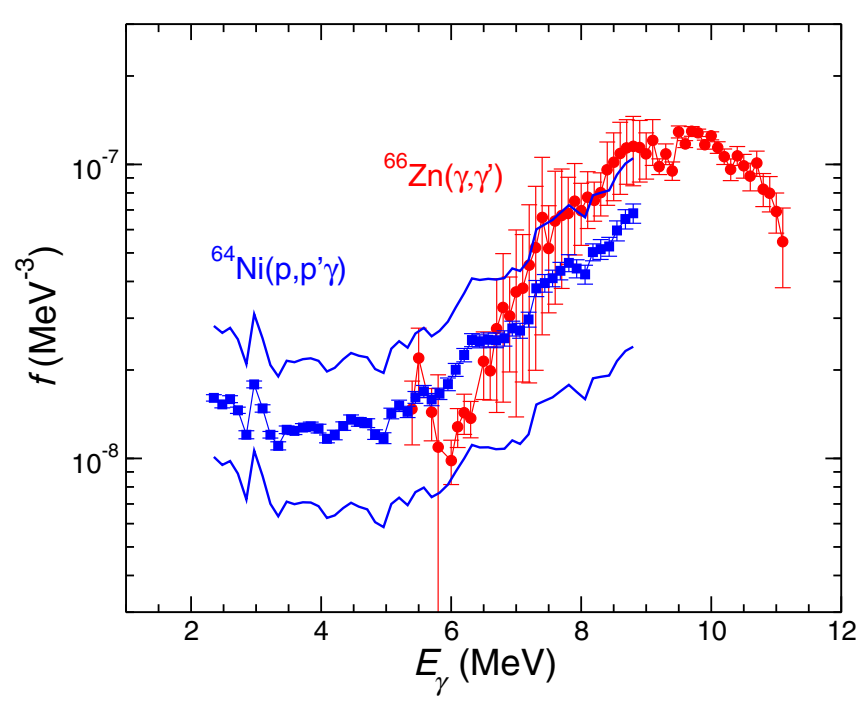

FIG. 9. Photon strength functions obtained from the present ${ }^{66} \mathrm{Zn}\left(\gamma, \gamma^{\prime}\right)$ data (red circles) and from ${ }^{64} \mathrm{Ni}\left(p, p^{\prime} \gamma\right)$ data taken from Ref. [90] (blue squares and lines).

found for ${ }^{54} \mathrm{Fe}$ [46] or other $N=28$ isotones [39-41,45], this shows that going from closed-shell to open-shell nuclides results in an increasing portion of $E 1$ strength and a decreasing portion of $M 1$ strength.

Reduced transition strengths $B(M 1)$ were deduced assuming branching ratios of $b_{0}=1$ for the ground-state transitions, because transitions from $J^{\pi}=1^{+}$states to the $2_{1}^{+}$state, which are expected to be the most intense branching transitions [84], were not identified. The experimental $B\left(M 1,1^{+} \rightarrow 0_{1}^{+}\right)$values are presented in Fig. 10.

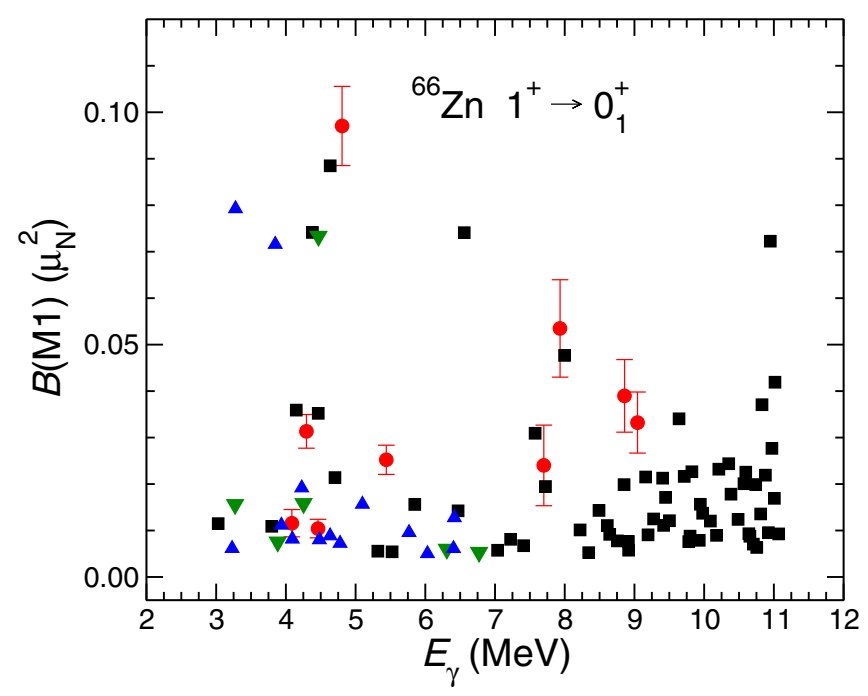

FIG. 10. Experimental $B\left(M 1,1^{+} \rightarrow 0_{1}^{+}\right)$values (red circles) and calculated values $B\left(M 1,1^{+} \rightarrow 0_{1}^{+}\right)>0.005 \mu_{N}^{2}$ from the lowest 150 $1^{+}$states (black squares). Also shown are values calculated with the same Hamiltonian (ca48mh1), but without proton excitations over the $Z=28$ shell gap (green downward triangles), and with the jj44c Hamiltonian (blue upward triangles). See text for details. 


\section{SHELL-MODEL CALCULATIONS}

Shell-model calculations for ${ }^{6} \mathrm{Zn}$ were carried out in the ca48pn model space with the ca48mh1 Hamiltonian [91] using the code NuSHELLX@MSU [92]. We note that this Hamiltonian was not specifically adjusted to nuclides around ${ }^{66} \mathrm{Zn}$. The model space included the $\pi\left(0 f_{7 / 2}^{(8-h)}, 0 f_{5 / 2}^{t}, 1 p_{3 / 2}^{t}, 1 p_{1 / 2}^{p 1}\right)$ proton orbits with $h=0$ to 1 , $t=0$ to $3, p 1=0$ to 2 , and the $v\left(0 f_{5 / 2}^{f 5}, 1 p_{3 / 2}^{p 3}, 1 p_{1 / 2}^{p 1}, 0 g_{9 / 2}^{g 9}\right)$ neutron orbits, where $f 5$ and $p 3$ can have values from zero to the respective maximum, and $g 9=0$ to 2 . The $2_{1}^{+}$state is calculated at $E_{x}=0.783 \mathrm{MeV}$ with a transition strength of $B\left(E 2,2_{1}^{+} \rightarrow 0_{1}^{+}\right)=14.8$ Weisskopf units (W.u.) using standard effective charges of $e_{\pi}=1.5 e$ and $e_{\nu}=0.5 e$. The corresponding experimental values are $E_{x}=1.039 \mathrm{MeV}$ and $B\left(E 2,2_{1}^{+} \rightarrow 0_{1}^{+}\right)=17.5$ W.u. Calculations with $h=0$ to 2 and $t=0$ to 4 in the given model space do not change these results and the results for the $1^{+}$states considerably.

Calculations for the lowest 150 states with $J^{\pi}=1^{+}$were performed and reduced transition strengths $B\left(M 1,1^{+} \rightarrow 0^{+}\right)$ were calculated using effective $g$ factors of $g_{s}^{\text {eff }}=0.74 g_{s}^{\text {free }}$. The calculated $B\left(M 1,1^{+} \rightarrow 0_{1}^{+}\right)$values are compared with the experimental ones deduced from the data in Table I in Fig. 10. Out of the 150 calculated values, only the 64 values greater than $0.005 \mu_{N}^{2}$ are displayed. This lower limit corresponds to the detection limit and is about half of the smallest experimental $B(M 1)$ value. The first calculated $1^{+}$state with a $B(M 1)$ value above this limit is the one at $3.029 \mathrm{MeV}$ with $B\left(M 1,1^{+} \rightarrow 0_{1}^{+}\right)=0.0115 \mu_{N}^{2}$, which is below the energy region investigated in the experiments at $\mathrm{HI} \gamma \mathrm{S}$. In the energy regions between about 4 and $5 \mathrm{MeV}$ and between 7 and $8 \mathrm{MeV}$, there are experimental and calculated values of similar magnitude up to about $0.1 \mu_{N}^{2}$ and additional weaker calculated values, whereas there are no experimental resolved transitions with strengths $B(M 1)>0.005 \mu_{N}^{2}$ between these regions. A possible reason for the differences is that a number of experimental $M 1$ transitions is below the detection limit, whereas the calculated ones are greater and therefore included in the figure. In other words, the calculations produced, in general, greater $B(M 1)$ values. Experimental strengths beyond $10 \mathrm{MeV}$ may be missing, because spin and parity assignments were not possible for the states above $10 \mathrm{MeV}$ (see Table I) and, therefore, they are not taken into account for the present comparison.

The running sums of the $B(M 1)$ values are graphed in Fig. 11. Both, the experimental and calculated curves show steps caused by great $B(M 1)$ values around $4.5 \mathrm{MeV}$ and additional ones at about $7.8 \mathrm{MeV}$ in the experimental and $6.5 \mathrm{MeV}$ in the calculated curve, followed by a more gradual increase of the latter. The calculated curve is by a factor of about 2 higher than the experimental one, caused by the greater calculated $B(M 1)$ values in the energy range between 4 and $5 \mathrm{MeV}$. One has to keep in mind that there may be experimental $B(M 1)$ strength below the detection limit in the quasicontinuum, whereas the corresponding calculated values exceed the lower limit of $0.005 \mu_{N}^{2}$ and appear as the many small values on the plateaus of the curve. Besides, some of the states between 10 and $11 \mathrm{MeV}$ in Table I may also be $1^{+}$ states with $B(M 1)$ values missing in the present experimental

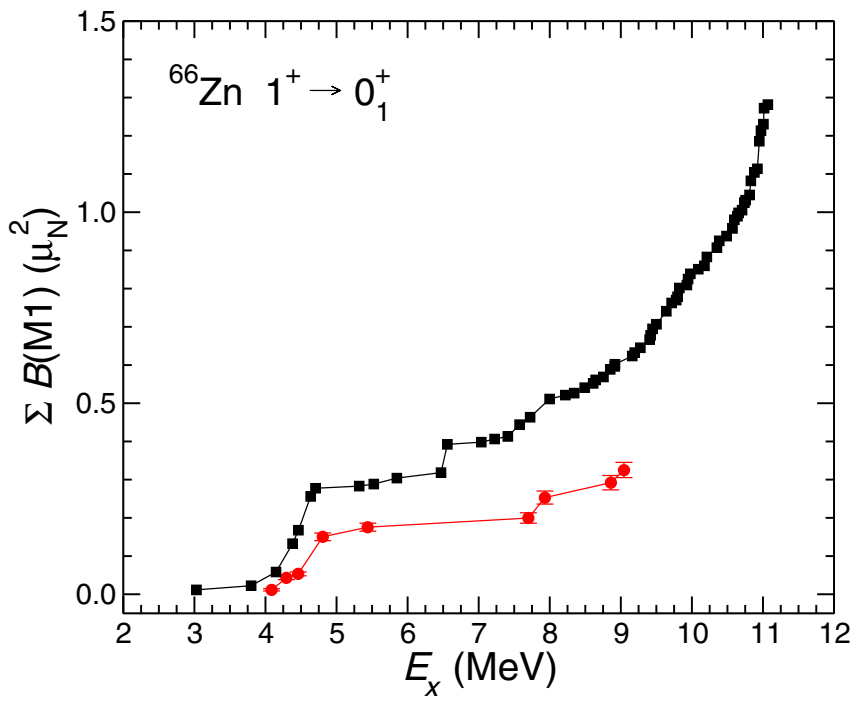

FIG. 11. Running sums $\sum B\left(M 1,1^{+} \rightarrow 0_{1}^{+}\right)$of the experimental (red circles) and calculated values (black squares) shown in Fig. 10.

sum. The summed calculated strength up to $E_{\gamma}=9.0 \mathrm{MeV}$ is $\sum B(M 1)_{\mathrm{cal}}=0.66 \mu_{N}^{2}$ and that of the values greater than $0.005 \mu_{N}^{2}$ only is $\sum B(M 1)_{\text {cal }}=0.60 \mu_{N}^{2}$, compared to an experimental sum of $\sum B(M 1)_{\exp }=0.33(2) \mu_{N}^{2}$.

The values in ${ }^{66} \mathrm{Zn}$ can be compared with the values $\sum B(M 1)_{\text {cal }}=1.76 \mu_{N}^{2}$ and $\sum B(M 1)_{\exp }=1.59(4) \mu_{N}^{2}$ for the range from $E_{\gamma}=6.8$ to $10.6 \mathrm{MeV}$ in ${ }^{54} \mathrm{Fe}$. The sum of all calculated strengths up to $11 \mathrm{MeV}$ in ${ }^{66} \mathrm{Zn}, \sum B(M 1)_{\mathrm{cal}}=$ $1.41 \mu_{N}^{2}$, amounts to about $64 \%$ of that in ${ }^{54} \mathrm{Fe}\left(\sum B(M 1)_{\mathrm{cal}}=\right.$ $\left.2.19 \mu_{N}^{2}\right)$ and supports the experimental fact that the $B(M 1)$ strength decreases when moving from $N=28$ into the open shell.

In addition to the calculations just described, the effects of specific configurations and alternative model spaces on the results were tested. Calculations not allowing proton excitations from the $0 f_{7 / 2}$ to the $\left(0 f_{5 / 2}, 1 p_{3 / 2}\right)$ orbits in the model space given above result in a few $B(M 1)$ values at low energy that are comparable to those of the more extended model space, but generally predict small $B(M 1)$ values $[B(M 1)<$ $\left.0.005 \mu_{N}^{2}\right]$, in particular at high energy, that are not incorporated in Fig. 10. Similar results were achieved in calculations using the jj44 model space and jj44c Hamiltonian [93,94]. The results with $B(M 1)>0.005 \mu_{N}^{2}$ are shown in Fig. 10 as well. These findings reveal that the proton excitations from the $0 f_{7 / 2}$ to the $\left(0 f_{5 / 2}, 1 p_{3 / 2}\right)$ orbits are the ones that generate the large M1 transition strengths, in particular for the high-lying states.

\section{CONCLUSIONS}

The dipole-strength distribution in ${ }^{66} \mathrm{Zn}$ was studied up to the neutron-separation energy in photon-scattering experiments at the $\gamma \mathrm{ELBE}$ and $\mathrm{HI} \gamma \mathrm{S}$ facilities using various electron energies. A total of 128 levels was identified. Spins $J=1$ were deduced from angular correlations of ground-state transitions measured at $\gamma$ ELBE and parities of states were determined from azimuthal asymmetries of intensities measured at $\mathrm{HI} \gamma \mathrm{S}$. The intensity distribution obtained from the measured spectra was corrected for the detector response. A 
simulated spectrum of photons scattered from the target by atomic interactions was subtracted. The remaining spectrum contains a continuum part in addition to the resolved peaks, which was included in the determination of the photoabsorption cross section. An assignment of inelastic transitions to particular levels and, thus, the determination of branching ratios was, in general, not possible. Therefore, simulations of statistical $\gamma$-ray cascades were performed to estimate intensities of branching transitions. These were subtracted from the experimental intensity distribution and the intensities of ground-state transitions were corrected on average for their branching ratios. In this way, a continuous photoabsorption cross section was derived for the energy range from about $5 \mathrm{MeV}$ up to the neutron threshold at $11.1 \mathrm{MeV}$, which can be combined with the $(\gamma, p)$ and $(\gamma, n)$ cross sections toward higher energies.

The absorption cross section of ${ }^{66} \mathrm{Zn}$ displays an extra strength on top of the tail of a Lorentz function for the GDR in the range between 6 and $11 \mathrm{MeV}$ that can be considered as the PDR. The shape of the PDR is smooth compared to the one in the nuclide ${ }^{54} \mathrm{Fe}$, which is characterized by several peaks caused by intense resolved transitions typical for the PDR in nuclides at closed-shells. The smooth PDR is well approximated by cross sections calculated in the statistical model as given in the TENDL library. The strength function deduced from the present ${ }^{66} \mathrm{Zn}$ data is similar to the one obtained from Oslo data for the neighboring isotone ${ }^{64} \mathrm{Ni}$. This shows, first, that the two methods produce roughly consistent results and, second, that the neighboring isotones have a similar structure.

The data indicate that the $M 1$ strength in ${ }^{66} \mathrm{Zn}$ amounts to about 5\% of the total strength in the resolved transitions. This represents a considerable reduction of $M 1$ strength compared to about $45 \%$ in ${ }^{54} \mathrm{Fe}$ and other nuclides around the shell closure at $N=28$. This means that the nuclear structure at closed shells induces strong $M 1,1^{+} \rightarrow 0_{1}^{+}$transitions, whereas the $M 1$ contribution to the strength function is small in open-shell nuclei.

The experimental $B(M 1)$ values were compared with predictions of shell-model calculations. The calculations yield in general greater $B(M 1)$ values compared with the experimental ones. There are, therefore, more calculated than observed transitions with $B(M 1)>0.005 \mu_{N}^{2}$ in the energy region under consideration. The experimental and calculated accumulative strengths differ by a factor of about 2 , but reveal similar characteristics, a step caused by large values between about 4 and $5 \mathrm{MeV}$, continued by a flat plateau up to $7 \mathrm{MeV}$ in the calculation and $8 \mathrm{MeV}$ in the experiment. Calculations in model spaces without proton excitations over the $Z=28$ gap are not capable of reproducing the experimental values, in particular not above about $5 \mathrm{MeV}$. This indicates the importance of those excitations for the generation of large $B(M 1)$ values in ${ }^{66} \mathrm{Zn}$.

\section{ACKNOWLEDGMENTS}

We thank the operating crews of the ELBE accelerator and of the TUNL storage ring and FEL for their cooperation and we thank J. Isaak and D. Savran for their help in setting up the experiment at $\mathrm{HI} \gamma \mathrm{S}$. Special thanks are due to B. A. Brown for his support in using the code NUSHELLX@MSU. This work was partially supported by the US Department of Energy (DOE), Office of Nuclear Physics, under Grants No. DEFG02-97ER41033 (TUNL) and No. DE-FG02-97ER41041 (UNC). R.M. acknowledges support by the US Department of Energy, Office of Science, Office of Nuclear Physics under Grant No. LANLEM77. The UWS group acknowledges financial support from UK STFC (Grant No. ST/P005101/1). J.S. acknowledges financial support by the UK Nuclear Data Network. T.B. and V.W. acknowledge support by the BMBF Grant No. 05P18RDEN9 and J. W. acknowledges support by the BMBF Grant No. 05P18PKEN9. T.B., U.F., J.K., and O.P. are supported by the Deutsche Forschungsgemeinschaft under Grant No. SFB 1245 (Project ID 279384907). This work was also supported by the grant "Nuclear Photonics" within the LOEWE program of the State of Hesse. R.S. gratefully acknowledges the allocation of computing time through the Centers for High-Performance Computing of Technische Universität Dresden and of Helmholtz-Zentrum Dresden-Rossendorf.
[1] S. Goriely et al., Eur. Phys. J. A 55, 172 (2019).

[2] T. Kawano et al., Nucl. Data Sheets 163, 109 (2020).

[3] W. Hauser and H. Feshbach, Phys. Rev. 87, 366 (1952).

[4] M. Arnould, S. Goriely, and K. Takahashi, Phys. Rep. 450, 97 (2007).

[5] F. Käppeler, R. Gallino, S. Bisterzo, and W. Aoki, Rev. Mod. Phys. 83, 157 (2011).

[6] U. Kneissl, N. Pietralla, and A. Zilges, J. Phys. G 32, R217 (2006).

[7] G. A. Bartholomew, E. D. Earle, A. J. Ferguson, J. W. Knowles, and M. A. Lone, in Advances in Nuclear Physics, edited by M. Baranger and E. Vogt, Vol. 7 (Springer, Boston, MA, 1973), pp. 229-324.

[8] D. Savran, T. Aumann, and A. Zilges, Prog. Part. Nucl. Phys. 70, 210 (2013).

[9] A. Bracco, E. G. Lanza, and A. Tamii, Prog. Part. Nucl. Phys. 106, 360 (2019).
[10] R. Massarczyk et al., Phys. Rev. Lett. 112, 072501 (2014).

[11] K. Heyde, P. von Neumann-Cosel, and A. Richter, Rev. Mod. Phys. 82, 2365 (2010).

[12] D. M. Brink, Ph.D. thesis, Oxford University, 1955 (unpublished).

[13] P. Axel, Phys. Rev. 126, 671 (1962).

[14] M. Guttormsen et al., Phys. Rev. Lett. 109, 162503 (2012)

[15] A. Simon et al., Phys. Rev. C 93, 034303 (2016).

[16] F. Naqvi, A. Simon, M. Guttormsen, R. Schwengner, S. Frauendorf, C. S. Reingold, J. T. Burke, N. Cooper, R. O. Hughes, S. Ota, and A. Saastamoinen, Phys. Rev. C 99, 054331 (2019).

[17] A. Voinov et al., Phys. Rev. Lett. 93, 142504 (2004).

[18] M. D. Jones et al., Phys. Rev. C 97, 024327 (2018).

[19] M. Guttormsen et al., Phys. Rev. C 71, 044307 (2005).

[20] A. C. Larsen et al., Phys. Rev. C 87, 014319 (2013). 
[21] R. Schwengner, S. Frauendorf, and A. C. Larsen, Phys. Rev. Lett. 111, 232504 (2013).

[22] B. A. Brown and A. C. Larsen, Phys. Rev. Lett. 113, 252502 (2014).

[23] R. Schwengner, S. Frauendorf, and B. A. Brown, Phys. Rev. Lett. 118, 092502 (2017).

[24] K. Sieja, Phys. Rev. Lett. 119, 052502 (2017).

[25] R. Schwengner and G. Rusev, Phys. Rev. C 100, 054320 (2019).

[26] R. Schwengner et al., Nucl. Instrum. Methods Phys. Res., Sect. A 555, 211 (2005).

[27] H. R. Weller, M. W. Ahmed, H. Gao, W. Tornow, Y. K. Wu, M. Gai, and R. Miskimen, Prog. Part. Nucl. Phys. 62, 257 (2009).

[28] A. Makinaga et al., Phys. Rev. C 94, 044304 (2016).

[29] R. Schwengner et al., Phys. Rev. C 87, 024306 (2013).

[30] G. Rusev et al., Phys. Rev. C 79, 061302(R) (2009).

[31] A. Makinaga et al., Phys. Rev. C 82, 024314 (2010).

[32] A. Makinaga et al., Phys. Rev. C 90, 044301 (2014).

[33] T. Shizuma et al., Phys. Rev. C 98, 064317 (2018).

[34] R. Schwengner et al., Phys. Rev. C 81, 054315 (2010).

[35] M. Beard, S. Frauendorf, B. Kämpfer, R. Schwengner, and M. Wiescher, Phys. Rev. C 85, 065808 (2012).

[36] N. Tsoneva, S. Goriely, H. Lenske, and R. Schwengner, Phys. Rev. C 91, 044318 (2015).

[37] R. Massarczyk, G. Rusev, R. Schwengner, F. Dönau, C. Bhatia, M. E. Gooden, J. H. Kelley, A. P. Tonchev, and W. Tornow, Phys. Rev. C 90, 054310 (2014).

[38] H. Pai et al., Phys. Rev. C 93, 014318 (2016).

[39] Krishichayan, M. Bhike, W. Tornow, G. Rusev, A. P. Tonchev, N. Tsoneva, and H. Lenske, Phys. Rev. C 91, 044328 (2015).

[40] T. Shizuma, T. Hayakawa, I. Daito, H. Ohgaki, S. Miyamoto, and F. Minato, Phys. Rev. C 96, 044316 (2017).

[41] J. Wilhelmy et al., Phys. Rev. C 98, 034315 (2018).

[42] F. Bauwens et al., Phys. Rev. C 62, 024302 (2000).

[43] T. Shizuma, T. Hayakawa, H. Ohgaki, H. Toyokawa, T. Komatsubara, N. Kikuzawa, T. Inakura, M. Honma, and H. Nakada, Phys. Rev. C 87, 024301 (2013).

[44] M. Scheck, V. Y. Ponomarev, T. Aumann, J. Beller, M. Fritzsche, J. Isaak, J. H. Kelley, E. Kwan, N. Pietralla, R. Raut, C. Romig, G. Rusev, D. Savran, K. Sonnabend, A. P. Tonchev, W. Tornow, H. R. Weller, and M. Zweidinger, Phys. Rev. C 87, 051304(R) (2013).

[45] M. Scheck et al., Phys. Rev. C 88, 044304 (2013).

[46] R. Schwengner et al., Phys. Rev. C 101, 064303 (2020).

[47] F. R. Metzger, Nucl. Phys. A 189, 409 (1972).

[48] D. C. Camp and G. L. Meredith, Nucl. Phys. A 166, 349 (1971).

[49] H. Verheul, Nucl. Data Sheets, Sec. B 2, 65 (1967).

[50] M. J. Martin and M. N. Rao, Nucl. Data Sheets, Sec. B 2, 43 (1968).

[51] N. Shikazono and Y. Kawarasaki, Nucl. Phys. A 118, 114 (1968).

[52] F. Ajzenberg-Selove, Nucl. Phys. A 506, 1 (1990).

[53] G. Rusev, A. P. Tonchev, R. Schwengner, C. Sun, W. Tornow, and Y. K. Wu, Phys. Rev. C 79, 047601 (2009).

[54] S. Agostinelli et al., Nucl. Instrum. Methods Phys. Res., Sect. A 506, 250 (2003).

[55] J. Allison et al., IEEE Trans. Nucl. Sci. 53, 270 (2006).

[56] J. Allison et al., Nucl. Instrum. Methods Phys. Res., Sect. A 835, 186 (2016).

[57] G. Rusev, Dissertation, Technische Universität Dresden 2007, Report FZD-478 (unpublished), http://www.hzdr.de/ publications/010008/10008.pdf
[58] R. Schwengner et al., Phys. Rev. C 76, 034321 (2007).

[59] G. Rusev et al., Phys. Rev. C 77, 064321 (2008).

[60] R. Massarczyk et al., Phys. Rev. C 86, 014319 (2012).

[61] M. Marta et al., Phys. Rev. C 81, 055807 (2010).

[62] E. Trompler, Diploma thesis, Technische Universität Dresden 2009, Report FZD-523 (unpublished), http://www.hzdr.de/ publications/013364/13364.pdf

[63] S. Carson, C. Iliadis, J. Cesaratto, A. Champagne, L. Downen, M. Ivanovic, J. Kelley, R. Longland, J. R. Newton, G. Rusev, and A. P. Tonchev, Nucl. Instrum. Methods Phys. Res., Sect. A 618, 190 (2010).

[64] E. Haug, Rad. Phys. Chem. 77, 207 (2008).

[65] G. Roche, C. Ducos, and J. Proriol, Phys. Rev. A 5, 2403 (1972).

[66] F. Salvat, J. D. Martinez, R. Mayol, and J. Parellada, Phys. Rev. A 36, 467 (1987).

[67] E. Browne and J. K. Tuli, Nucl. Data Sheets 111, 1093 (2010).

[68] R. Schwengner et al., Phys. Rev. C 78, 064314 (2008).

[69] N. Benouaret et al., Phys. Rev. C 79, 014303 (2009).

[70] N. Pietralla, Z. Berant, V. N. Litvinenko, S. Hartman, F. F. Mikhailov, I. V. Pinayev, G. Swift, M. W. Ahmed, J. H. Kelley, S. O. Nelson, R. Prior, K. Sabourov, A. P. Tonchev, and H. R. Weller, Phys. Rev. Lett. 88, 012502 (2001).

[71] A. K. Furr, E. L. Robinson, and C. H. Robins, Nucl. Instrum. Methods 63, 205 (1968).

[72] R. Massarczyk et al., Phys. Rev. C 92, 044309 (2015).

[73] G. Schramm et al., Phys. Rev. C 85, 014311 (2012).

[74] F. Bečvář, Nucl. Instrum. Methods Phys. Res., Sect. A 417, 434 (1998).

[75] C. E. Porter and R. G. Thomas, Phys. Rev. 104, 483 (1956).

[76] A. Gilbert and A. G. W. Cameron, Can. J. Phys. 43, 1446 (1965).

[77] T. von Egidy and D. Bucurescu, Phys. Rev. C 80, 054310 (2009).

[78] S. I. Al-Quraishi, S. M. Grimes, T. N. Massey, and D. A. Resler, Phys. Rev. C 67, 015803 (2003).

[79] A. R. Junghans, G. Rusev, R. Schwengner, A. Wagner, and E. Grosse, Phys. Lett. B 670, 200 (2008).

[80] E. Grosse, A. R. Junghans, and R. Massarczyk, Eur. Phys. J. A 53, 225 (2017).

[81] S. Raman, C. W. Nestor Jr., and P. Tikkanen, At. Data Nucl. Data Tables 78, 128 (2001).

[82] J.-P. Delaroche, M. Girod, J. Libert, H. Goutte, S. Hilaire, S. Péru, N. Pillet, and G. F. Bertsch, Phys. Rev. C 81, 014303 (2010).

[83] R. Capote et al., Nucl. Data Sheets 110, 3107 (2009).

[84] A. P. Tonchev, S. L. Hammond, J. H. Kelley, E. Kwan, H. Lenske, G. Rusev, W. Tornow, and N. Tsoneva, Phys. Rev. Lett. 104, 072501 (2010).

[85] J. Wilhelmy et al., Phys. Rev. C 102, 044327 (2020).

[86] S. Costa, F. Ferrero, C. Manfredotti, L. Pasqualini, G. Piragino, and H. Arenhövel, Nuovo Cimento B 48, 460 (1967).

[87] A. J. Koning, D. Rochman, J. Sublet, N. Dzysiuk, M. Fleming, and S. van der Marck, Nucl. Data Sheets 155, 1 (2019).

[88] A. Koning (private communication).

[89] M. Guttormsen, T. S. Tveter, L. Bergholt, F. Ingebretsen, and J. Rekstad, Nucl. Instrum. Methods Phys. Res., ect. A 374, 371 (1996).

[90] L. Crespo Campo, F. L. Bello Garrote, T. K. Eriksen, A. Gorgen, M. Guttormsen, K. Hadynska-Klek, M. Klintefjord, 
A. C. Larsen, T. Renstrom, E. Sahin, S. Siem, A. Springer, T. G. Tornyi, and G. M. Tveten, Phys. Rev. C 94, 044321 (2016).

[91] M. Hjorth-Jensen, T. T. S. Kuo, and E. Osnes, Phys. Rep. 261, 125 (1995), and M. Hjorth-Jensen (private communication).

[92] B. A. Brown and W. D. M. Rae, Nucl. Data Sheets 120, 115 (2014).
[93] A. F. Lisetskiy, B. A. Brown, M. Horoi, and H. Grawe, Phys. Rev. C 70, 044314 (2004).

[94] S. Mukhopadhyay, B. P. Crider, B. A. Brown, S. F. Ashley, A. Chakraborty, A. Kumar, M. T. McEllistrem, E. E. Peters, F. M. Prados-Estévez, and S. W. Yates, Phys. Rev. C 95, 014327 (2017). 